\title{
Strain in crystalline core-shell nanowires
}

\author{
David Ferrand ${ }^{1}$ and Joël Cibert ${ }^{2, a}$ \\ ${ }^{1}$ Université Grenoble Alpes, Inst NEEL, 38042 Grenoble, France \\ 2 CNRS, Inst NEEL, 38042 Grenoble, France
}

Received: 14 April 2014 / Received in final form: 25 June 2014 / Accepted: 30 June 2014 Published online: 20 August 2014 - (c) The Author(s) 2014

\begin{abstract}
We propose a comprehensive description of the strain configuration induced by the lattice mismatch in a core-shell nanowire with circular cross-section, taking into account the crystal anisotropy and the difference in stiffness constants of the two materials. We use an analytical approach which fully exploits the symmetry properties of the system. Explicit formulae are given for nanowires with the wurtzite structure or the zinc-blende structure with the hexagonal/trigonal axis along the nanowire, and the results are compared to available numerical calculations and experimental data on nanowires made of different III-V and II-VI semiconductors. The method is also applied to multishell nanowires, and to core-shell nanowires grown along the $\langle 001\rangle$ axis of cubic semiconductors. It can be extended to other orientations and other crystal structures.
\end{abstract}

\section{Introduction}

Semiconductor nanowires (NWs) are often grown in the form of core-shell structures, in order to achieve better photonic and electronic properties: the active core is isolated from the surface defects and traps in order to obtain a better luminescence efficiency, sharper linewidths, longer coherence times and higher mobility, or even a better chemical stability. As the lattice parameter of the shell is generally different from that of the core, and since coherent structures are contemplated with no misfit defects at the interface, the elastic strain induced in the core and its effect on the electronic properties have to be taken into account. In turn, the built-in strain can be used as a further adjustable parameter: strain engineering can be used to lower the degeneracy in the valence band and select the type of holes with a larger spin for spintronics applications (for instance a larger spin-carrier coupling in diluted magnetic semiconductors) [1], or a smaller longitudinal mass to achieve a better mobility in transport properties [2]. The strain can also be designed to induce a built-in piezoelectric field, resulting in a faster separation of the electron-hole pairs in photovoltaic applications [3]. Finally, strain is an important parameter when engineering Si-Ge NWs to obtain direct bandgap configurations and efficient emission of light [4].

Analytical expressions exist for a core-shell structure made of elastically isotropic materials [5]. However, the crystal structure results in anisotropic elastic properties, the core and shell materials have different values of the stiffness constants, and the NW shape can deviate from

\footnotetext{
a e-mail: joel.cibert@neel.cnrs.fr
}

the ideal cylinder with a circular base and for instance feature facetting. As a result, calculating the strain configuration in a real semiconductor core-shell NW is not an easy task: quantitative descriptions usually imply to compute numerically the local strain, either using a microscopic model such as the valence force field model, or performing a finite element treatment of the continuum elasticity theory [6]. Nevertheless, an analytical description, such as what has been developed and reviewed in reference [7] for the case of quantum dots and NWs embedded in an infinite or semi-infinite material, remains the best starting point for an implementation of the strainrelated mechanisms governing the electronic properties, through deformation potentials and piezoelectric fields.

A quantitative, fully analytical solution for core-shell NWs, taking into account the crystal structure, can be found, and this is the purpose of the present study. Starting with the well-known expression for isotropic materials (and their extension for the transversely isotropic materials), we propose solutions for the most often encountered cases of zinc-blende and wurtzite semiconductors. We give analytical expressions for the strain in the core and in the shell, and for their effect on the extrema of bands, and we compare these predictions to the results of microscopic calculations and experimental data.

In most cases, deviations from the cylindrical strain configuration are found. In two typical cases (with zincblende or diamond semiconductor NWs along $\langle 111\rangle$ and along $\left\langle\begin{array}{lll}0 & 0 & 1\rangle\end{array}\right\rangle$ ), we identify the resulting strain configuration to first-order in the parameter describing the cubic anisotropy and we show that these deviations from cylindrical symmetry are rather small. These two cases 
illustrate two non-isotropic strain configurations: warping along the NW axis, and anisotropy of the in-plane strain. Other orientations and crystal structures are expected to feature a combination of these two configurations.

To sum up our results: (i) the cylindrical approximation is surprisingly good, provided one uses the appropriate truncation of the stiffness tensor which is given here; (ii) the result is exact for wurtzite NWs grown along the hexagonal axis; (iii) a first-order treatment of the anisotropy quantitatively agrees with available numerical results for zinc-blende NWs; the additional strain components are negligible in the core but they take significant values in the shell; (iii) the present method is readily extended to other structures or orientations, and to multishell NWs.

The paper is organized as follows: Section 2 is a short summary of the problem to be solved and of results which are well-known for isotropic materials. In Section 3, we obtain analytical expressions of the strain configuration in wurtzite semiconductor NWs grown along the hexagonal axis; the transfer matrix approach allows us to consider both core-shell and multishell NWs. In Sections 4 and 5, we use a perturbation method to describe the more complex strain configuration present in zinc-blende semiconductor NWs grown along the trigonal axis and along the cubic axis.

\section{Strain and electrons in a core-shell NW}

\subsection{The displacement field in an infinite core-shell NW}

In this section we recall the well-known strain configuration in an infinitely long core-shell NW with circular crosssection, made of isotropic materials, in order to identify and illustrate the effects of the two elements of symmetry on the displacement field and the Lamé-Clapeyron-Navier equation. We consider a cylinder-shaped core (superscript or subscript $c$ ), infinitely long, with a circular cross-section of radius $r_{c}$, embedded in a shell (superscript or subscript $s$ ) of radius $r_{s}$. We note $z$ the NW axis, $(r, \theta)$ or $(x, y)$ the in-plane coordinates measured from the NW axis. The two materials have the same crystal structure and the same orientation, with different values of the lattice constants $a_{s}$ and $a_{c}$. The growth is assumed to be coherent, with no misfit defect at the interface, so that the lattice mismatch $f=\left(a_{s}-a_{c}\right) / a_{c}$ is fully accommodated by elastic strain.

The general solution involves calculating the displacement field $\mathbf{u}(\mathbf{r})$ which relates the position of any point $\mathbf{r}$ in the strained material to its value in the mismatched, unstrained system. The local deformation, in the vicinity of a point $\mathbf{r}$, is fully described by the tensor of the derivatives of $\mathbf{u}(\mathbf{r}), \partial u_{i} / \partial x_{j}$ : the symmetric part is the strain tensor, $\varepsilon_{i j}=\frac{1}{2}\left(\partial u_{i} / \partial x_{j}+\partial u_{j} / \partial x_{i}\right)$, associated to elastic energy, while the antisymmetric part $\frac{1}{2}\left(\partial u_{i} / \partial x_{j}-\right.$ $\left.\partial u_{j} / \partial x_{i}\right)$ describes a local rotation. In the presence of body forces per unit volume $\mathbf{F}(\mathbf{r})$, the equilibrium condition, $\sum_{j} \partial \sigma_{i j} / \partial x_{j}+F_{i}=0$, can be expressed as the Lamé-Clapeyron-Navier equation (there is one equation for each value of $i$ and $\left.x_{i}=x, y, z\right)$,

$$
\sum_{j k l} c_{i j k l} \frac{\partial}{\partial x_{j}}\left(\frac{\partial u_{k}}{\partial x_{l}}+\frac{\partial u_{l}}{\partial x_{k}}\right)+F_{i}=0 .
$$

In this equation, the $c_{i j k l}$ are the components of the stiffness tensor, which relates the stress tensor $\sigma_{i j}$ to the strain tensor $\varepsilon_{k l}$ through the Hooke's law, $\sigma_{i j}=\sum_{k l} c_{i j k l} \varepsilon_{k l}$. The number of independent components $c_{i j k l}$ is determined by the symmetry properties of the material [8].

In a core-shell NW, we apply the Lamé-ClapeyronNavier equation within each constituent; the body forces are zero, but we have to apply proper boundary conditions [9] at the surface and at the interface. A first series of conditions ensure the stability of the interface/surface: stress components applied to the surface $\left(\sigma_{r r}, \sigma_{r \theta}\right.$ and $\left.\sigma_{r z}\right)$ vanish, and they are equal on both sides of the interface. Additional conditions state the continuity of the lattice: the displacement field $\mathbf{u}(\mathbf{r})$ must compensate for the lattice mismatch $f$. All these conditions are actually the same as for a thin epitaxial layer, but then the condition on the continuity of the lattice can be expressed on the in-plane strain components [10].

In addition, for an infinitely long NW, the overall translational invariance along the axis must be maintained (and it is known also that in a NW of finite length, according to the Saint-Venant's principle, this holds everywhere but for a segment of length equal to about the diameter at each end). Translational invariance means that the relative displacement of two neighboring points is independent of $z$, i.e., that all derivatives of $\mathbf{u}(\mathbf{r})$ are independent of $z: \partial / \partial z\left(\partial u_{i} / \partial x_{j}\right)=0$, or $\partial / \partial x_{j}\left(\partial u_{i} / \partial z\right)=0$, hence $\partial u_{i} / \partial z$ is a constant $C_{i}$ independent of $\mathbf{r}, \partial u_{i} / \partial z=C_{i}$, and $u_{i}(\mathbf{r})=C_{i} z+D+u_{i}(x, y)$. Note that the $C_{x} z, C_{y} z$ and $u_{z}(x, y)$ contributions correspond to shear strains $\left(\varepsilon_{x z}\right.$, $\left.\varepsilon_{y z}\right)$ and are often excluded by symmetry. Finally, the equilibrium with respect to a translation along the NW axis requires that the longitudinal stress integrated over the NW section be zero.

Once determined the displacement field $\mathbf{u}(\mathbf{r})$ obeying the Lamé-Clapeyron-Navier equation and the boundary conditions, the strain tensor can be introduced into the socalled deformation potentials [11] and the possible piezoelectric field is calculated; the positions of the conduction and valence band edges follow.

\subsection{The simple case of elastically isotropic materials}

The solution for an infinitely long, circular core-shell structure made of elastically isotropic materials, is well known [5]. We briefly recall the main results, our goal being to examine what will remain valid if materials with a lower symmetry are involved.

The Lamé-Clapeyron-Navier equation writes:

$$
\mu \sum_{j} \frac{\partial^{2} u_{i}}{\partial x_{j}^{2}}+(\lambda+\mu) \sum_{j} \frac{\partial^{2} u_{j}}{\partial x_{i} \partial x_{j}}=0,
$$

which contains three equations, for $x_{i}=x, y$ and $z$, respectively. A more compact form better evidences the spherical 
symmetry:

$$
\mu \Delta \mathbf{u}+(\lambda+\mu) \nabla(\nabla \cdot \mathbf{u})=\mathbf{0} .
$$

Here the stiffness tensor has only two independent components: the so-called Lamé coefficients $\mu=c_{i j i j}=c_{i j j i}$ and $\lambda=c_{i i j j}$ for $i \neq j$, with $c_{i i i i}=\lambda+2 \mu$. All other components vanish.

If we omit the terms which vanish due to the invariance by translation or would correspond to axial shear strains (according to the discussion in the previous section), the Lamé-Clapeyron-Navier equation restricts to:

$$
\begin{aligned}
& \mu\left(\frac{\partial^{2}}{\partial y^{2}}+\frac{\partial^{2}}{\partial x^{2}}\right) u_{x}+(\lambda+\mu) \frac{\partial}{\partial x}\left(\frac{\partial u_{x}}{\partial x}+\frac{\partial u_{y}}{\partial y}\right)=0 \\
& \mu\left(\frac{\partial^{2}}{\partial y^{2}}+\frac{\partial^{2}}{\partial x^{2}}\right) u_{y}+(\lambda+\mu) \frac{\partial}{\partial y}\left(\frac{\partial u_{x}}{\partial x}+\frac{\partial u_{y}}{\partial y}\right)=0 \\
& \mu\left(\frac{\partial^{2}}{\partial y^{2}}+\frac{\partial^{2}}{\partial x^{2}}\right) u_{z}=0 .
\end{aligned}
$$

As the strained system obviously retains the cylindrical symmetry, we write the displacement field in cylindrical coordinates, keeping only the relevant variables: $u_{r}(r)$, $u_{\theta}=0$, with $u_{x}=u_{r}(r) \cos \theta$ and $u_{y}=u_{r}(r) \sin \theta$. An in-plane dependence of $u_{z}$ would imply shear strain components $\varepsilon_{r z}$ which are excluded, hence $u_{z}(z)=$ $C z+D$. Finally the Lamé-Clapeyron-Navier equation is reduced to equating to zero the Laplacian of the in-plane displacement, hence $\mathrm{d}^{2} u_{r} / \mathrm{d} r^{2}+\mathrm{d} u_{r} / r \mathrm{~d} r-u_{r} / r^{2}=0$, and $u_{r}(r)=A r+B r_{c}^{2} / r$, with parameters $A, B, C$ and $D$ to be determined in each material. The non-vanishing components of the strain tensor are thus the longitudinal expansion $\varepsilon_{z z}=\mathrm{d} u_{z} / \mathrm{d} z=C$, the radial expansion $\varepsilon_{r r}=\mathrm{d} u_{r} / \mathrm{d} r=A-B r_{c}^{2} / r^{2}$, and the angular expansion $\varepsilon_{\theta \theta}=u_{r} / r=A+B r_{c}^{2} / r^{2}$. Note that $B$ vanishes in the core (to avoid diverging terms at the axis, $r=0$ ); also, $D$ represents a global displacement of the core or the shell, hence $D=0$. As a result, see Figure 1, the strain (and the stress) are uniform in the core; in the shell, there is also a uniform component, and a non-uniform shear component, rotating around the interface and close to it. Note that the stress component $\sigma_{z z}^{c}$ is uniform also in the shell (the non-uniform $B r_{c}^{2} / r^{2}$ terms in $\varepsilon_{\theta \theta}^{s}$ and $\varepsilon_{r r}^{s}$ cancel each other when applying the Hooke's law).

The two parameters $A_{c}$ and $C_{c}$ in the core, and the three parameters $A_{s}, B_{s}$ and $C_{s}$ in the shell, are determined from the boundary conditions. At the interface, the matching along $z$ (written on $u_{z}$ or $\varepsilon_{z z}$ ) implies $C^{c}-C^{s}=$ $f_{\|}$, and the matching in the plane is realized simultaneously on $u_{r}$ and $\varepsilon_{\theta \theta}$ if $A^{c}-A^{s}-B^{s}=f_{\perp}$. We identify the mismatch $f_{\|}$in the direction of the NW axis, and the mismatch $f_{\perp}$ in the plane perpendicular to the axis: although this is not done usually - and not needed for isotropic materials - that will allow a better understanding of the result. The stress components are such that $\sigma_{r r}^{c}\left(r_{c}\right)-\sigma_{r r}^{s}\left(r_{c}\right)=0$ at the interface and $\sigma_{r r}^{s}\left(r_{s}\right)=0$ at the sidewall. The other components $\left(\sigma_{r \theta}\right.$ and $\left.\sigma_{r z}\right)$ automatically vanish. The longitudinal stress integrated over the NW section vanishes: as both $\sigma_{z z}^{s}$ and $\sigma_{z z}^{c}$ are uniform, the condition is simply $\eta \sigma_{z z}^{c}+(1-\eta) \sigma_{z z}^{s}=0$ where $\eta$ is the ratio of the core to NW cross-section areas (for a NW with circular cross-section, $\eta=r_{c}^{2} / r_{s}^{2}$ ).

A straightforward calculation then gives the complete set of strain components:

$$
\begin{aligned}
\varepsilon_{z z}^{c} & =(1-\eta) f_{\|} \\
\varepsilon_{z z}^{s} & =-\eta f_{\|} \\
\frac{\varepsilon_{\theta \theta}^{s}-\varepsilon_{r r}^{s}}{2} & =B_{s} \frac{r_{c}^{2}}{r^{2}} \\
\varepsilon_{\theta \theta}^{c}=\varepsilon_{r r}^{c} & =(1-\eta)\left(f_{\perp}+B_{s}\right) \\
\frac{\varepsilon_{\theta \theta}^{s}+\varepsilon_{r r}^{s}}{2} & =-\eta\left(f_{\perp}+B_{s}\right),
\end{aligned}
$$

where

$$
\begin{aligned}
B_{s} & =-\frac{2(\lambda+\mu) f_{\perp}+\lambda f_{\|}}{2(\lambda+2 \mu)} \\
\left(f_{\perp}+B_{s}\right) & =\frac{2 \mu f_{\perp}-\lambda f_{\|}}{2(\lambda+2 \mu)} .
\end{aligned}
$$

The longitudinal strain $\varepsilon_{z z}$ (red arrows in Fig. 1) results from the lattice mismatch in the direction of the NW axis, which is shared between the core and the shell with a weight inversely proportional to their area (in a way similar to the strain distribution in a free-standing superlattice, where the lattice mismatch is shared with a weight inversely proportional to the thickness of each layer). A narrow core is fully strained to the thick shell (and a thin shell to a wide core). The main part of the in-plane lattice mismatch is accommodated by the shear strain in the shell rotating around the interface (green arrows in Fig. 1). The rest of the in-plane strain consists in a uniform inplane strain in the core and a uniform component in the shell (blue arrows in Fig. 1): these components result from the competition between a direct effect of the lattice mismatch in the plane, and an indirect effect of the longitudinal strain. As a result, they can be quite small.

For a thin shell, $\eta=1$, the core is unstrained, and the shell strain writes $\varepsilon_{z z}^{s}=\varepsilon_{\theta \theta}^{s}=-f, \varepsilon_{r r}^{s}=[2 \lambda /(\lambda+2 \mu)] f$, which is the result for a thin epitaxial layer on a plane substrate.

The previous result can be extended [12] to the case of two isotropic materials with different values of the shear modulus, but the same value of the Poisson ratio. In terms of Lamé coefficients, that means $\lambda_{s} / \lambda_{c}=\mu_{s} / \mu_{c}$. Complete expressions of the stress tensor are given in reference [12]. We will generalize these expressions in the following section taking into account the crystal structure.

\subsection{The effect on the electronic properties}

Two mechanisms affecting the electronic properties of a core-shell NW are determined by the strain configuration.

- there is a direct effect of strain on the bands of a semiconductor; around the band edges, it is described phenomenologically by the so-called deformation potentials. For instance, in a zinc-blende semiconductor, 


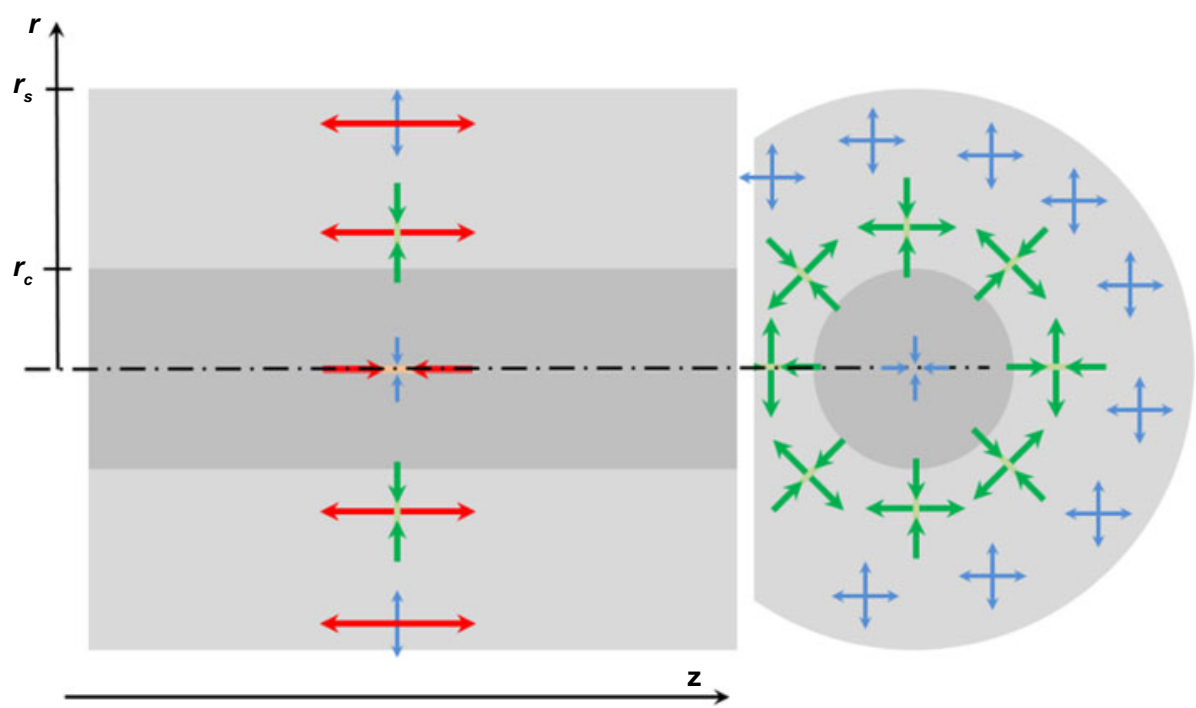

Fig. 1. Strain distribution in a cylindrical core-shell NW. Arrows indicate the longitudinal strain (in red), the inhomogeneous shear strain in the shell (in green), and the rest of the strain - uniform and isotropic in the plane (in blue). The lattice parameter is assumed to be smaller in the shell than in the core $(f<0)$.

the isotropic strain (change of volume), $\left(\varepsilon_{x x}+\varepsilon_{y y}+\right.$ $\varepsilon_{z z}$ ), induces a shift of the conduction band and an average shift of the valence band at the center of the Brillouin zone. A shear strain, such as $\left(2 \varepsilon_{z z}-\varepsilon_{x x}-\varepsilon_{y y}\right)$ induces a splitting of the valence band edge;

- when NWs are grown along a polar axis, they are expected to present a polarization due to the piezoelectric effect. This is the case of NWs with the wurtzite structure grown along the $c$-axis, as well as NWs with the zinc-blende structure grown along the $\langle 111\rangle$ axis. The relevant strain components entering the longitudinal polarization are [3] $\varepsilon_{z z}$ and $\left(\varepsilon_{r r}+\varepsilon_{\theta \theta}\right)$ in the first case, and $\left(2 \varepsilon_{z z}-\varepsilon_{x x}-\varepsilon_{y y}\right)$ in the second case.

In addition, confinement effects should be taken into account if the NW radius is small enough, and the confining potential is modified by these two mechanisms.

It is interesting to compare the results for a thin core and that for a thin epitaxial layer, both considered as the active medium of the structure. In both cases there is an isotropic strain and a shear strain. The isotropic strain is $\left(\varepsilon_{x x}+\varepsilon_{y y}+\varepsilon_{z z}\right)$ or $\left(\varepsilon_{r r}+\varepsilon_{\theta \theta}+\varepsilon_{z z}\right)=[4 \mu /(\lambda+2 \mu)] f$ in both cases. The shear strain is $\left(2 \varepsilon_{z z}-\varepsilon_{x x}-\varepsilon_{y y}\right)=$ $-2[(3 \lambda+2 \mu) /(\lambda+2 \mu)] f$ in the thin epitaxial layer, and $\left(2 \varepsilon_{z z}-\varepsilon_{r r}-\varepsilon_{\theta \theta}\right)=[(3 \lambda+2 \mu) /(\lambda+2 \mu)] f$. The same result holds for a thick core, with $f$ replaced by $\eta f$. Hence the ratio of the valence band splitting to the shift is (1) of opposite sign, and (2) twice smaller, in the core of a NW than in an epitaxial layer made of elastically isotropic materials. This property will be checked below in the presence of crystalline anisotropy: we will show that the factor is not exactly two.

\subsection{Crystalline semiconductor NWs}

Our goal is to take into account the crystal structure of the semiconductors, by using the stiffness tensor with the appropriate symmetry. We will consider explicitly three cases: hexagonal (wurtzite) structure with the NW axis along the $c$-axis, and cubic (zinc-blende or diamond) structure with the NW axis along $\langle 001\rangle$ or $\langle 111\rangle$. We ignore facetting and consider a NW with a circular cylinder shape. We will show that:

- in the case of a wurtzite NW grown along the six-fold axis, the transversely isotropic solution is exact;

- in the case of a zinc-blende NW grown along a trigonal axis, the transversely isotropic solution is an excellent approximation, which reproduces quantitatively the results of numerical approaches. Deviations due to the cubic anisotropy appear in the form of a warping along the axis, of three-fold symmetry, and can be found as the response of an elastically isotropic system to a distribution of body forces parallel to the NW axis;

- in the case of a zinc-blende NW grown along a tetragonal axis, a transversely isotropic approximation is proposed. Deviations with four-fold symmetry are found and calculated as the response to a distribution of body forces perpendicular to the NW axis.

The stiffness tensor is written using the Voigt notation, $\varepsilon_{1}=\varepsilon_{x x}, \ldots, \varepsilon_{4}=\varepsilon_{y z}+\varepsilon_{z y}, \ldots$, and $c_{x x x x}=c_{11}, c_{z z z z}=$ $c_{33}, c_{y z y z}=c_{44}, c_{x y x y}=c_{66}$ and so on.

The stiffness tensor for the zinc-blende structure reflects the cubic symmetry [8]. It contains three independent terms and the Voigt notation in the cubic axes is:

$$
\left(\begin{array}{cccccc}
c_{11} & c_{12} & c_{12} & 0 & 0 & 0 \\
c_{12} & c_{11} & c_{12} & 0 & 0 & 0 \\
c_{12} & c_{12} & c_{11} & 0 & 0 & 0 \\
0 & 0 & 0 & c_{44} & 0 & 0 \\
0 & 0 & 0 & 0 & c_{44} & 0 \\
0 & 0 & 0 & 0 & 0 & c_{44}
\end{array}\right)
$$

The anisotropy is characterized by the parameter $c=$ $\left(c_{11}-c_{12}-2 c_{44}\right)$. If $c=0$, the energy of a tetragonal shear 
strain (characterized by $c_{11}-c_{12}$ ) equals that of a trigonal shear strain (characterized by $2 c_{44}$ ) and the spherical symmetry is restored. Usual semiconductors have $c<0$ : they are harder against a trigonal stress, which directly involves a change of bond length, than against a tetragonal stress which is accommodated mainly by bond rotation. As a result, they are harder along a $\langle 111\rangle$ direction and softer along a $\langle 001\rangle$ direction, with $\langle 110\rangle$ in between [13].

In the wurtzite structure, with $z$ along the $c$-axis and $x, y$ in the perpendicular plane, symmetry considerations imply identities such as $c_{22}=c_{11}$ or $c_{66}=\left(c_{11}-c_{12}\right) / 2$, so that the stiffness tensor has five independent components $[8]$ :

$$
\left(\begin{array}{cccccc}
c_{11} & c_{12} & c_{13} & 0 & 0 & 0 \\
c_{12} & c_{11} & c_{13} & 0 & 0 & 0 \\
c_{13} & c_{13} & c_{33} & 0 & 0 & 0 \\
0 & 0 & 0 & c_{44} & 0 & 0 \\
0 & 0 & 0 & 0 & c_{44} & 0 \\
0 & 0 & 0 & 0 & 0 & \frac{c_{11}-c_{12}}{2}
\end{array}\right)
$$

It is invariant under any rotation around the $c$-axis.

\section{Hexagonal semiconductors along the c-axis}

We consider a NW with the wurtzite structure, and its axis parallel to the $c$ axis. We take the $z$ axis along this axis, and $x$ and $y$ two arbitrary axes in the basal plane. Note that the lattice mismatch along the $c$-axis, $f_{\|}$, and perpendicular to it, $f_{\perp}$, may be different.

\subsection{Calculation}

Our calculation is similar to that of reference [14], where the stress is calculated for coaxial cylinders with transverse isotropy: indeed this is the case for hexagonal semiconductors around the $c$-axis. In this section, we give the full expressions of the strain, which are the useful parameters to calculate the local potential and the piezoelectric field. Moreover, this part constitutes our first step for the calculation of strain in systems lacking transverse isotropy.

The complete Lamé-Clapeyron-Navier equation (Eq. (1)) is written in Appendix A (Eq. (A.1)). Omitting terms which vanish due to invariance by translation, we obtain:

$$
\begin{aligned}
& \frac{c_{11}-c_{12}}{2}\left(\frac{\partial^{2}}{\partial y^{2}}+\frac{\partial^{2}}{\partial x^{2}}\right) u_{x} \\
& \quad+\frac{c_{11}+c_{12}}{2} \frac{\partial}{\partial x}\left(\frac{\partial u_{x}}{\partial x}+\frac{\partial u_{y}}{\partial y}\right)=0 \\
& \frac{c_{11}-c_{12}}{2}\left(\frac{\partial^{2}}{\partial y^{2}}+\frac{\partial^{2}}{\partial x^{2}}\right) u_{y} \\
& \quad+\frac{c_{11}+c_{12}}{2} \frac{\partial}{\partial y}\left(\frac{\partial u_{x}}{\partial x}+\frac{\partial u_{y}}{\partial y}\right)=0 \\
& \left(\frac{\partial^{2}}{\partial y^{2}}+\frac{\partial^{2}}{\partial x^{2}}\right) u_{z}=0 .
\end{aligned}
$$

It reproduces exactly the Lamé-Clapeyron-Navier equation of an elastically isotropic material, equation (4). Hence, as the boundary conditions are invariant under a rotation around the NW axis (this is due to the invariance of the stiffness tensor noted above), the general solution for equation (9) is the same as that of equation (4), $u_{z}(z)=C z$ and $u_{r}(r)=A r+B r_{c}^{2} / r$. Furthermore, as this solution is such that the terms of equation (A.1) omitted in equation (9) all vanish, it is the exact solution of the complete equation, equation (A.1).

Applying the Hooke's law to the boundary conditions of a core-shell NW as in the previous section (at the interface, step in $u_{z} / z=\varepsilon_{z z}$ and in $u_{r} / r=\varepsilon_{\theta \theta}$ to accommodate the lattice mismatch $f_{\|}$and $f_{\perp}$ with no misfit dislocation, and equilibrium of $\sigma_{r r}$; at the sidewall, $\sigma_{r r}^{s}\left(r_{s}\right)=0$; along the $z$-axis, $\left.\eta \sigma_{z z}^{c}+(1-\eta) \sigma_{z z}^{s}=0\right)$, we obtain the strain tensor by inverting a system of linear equations:

$$
\begin{gathered}
\left(\begin{array}{ccccc}
0 & 0 & 0 & 1 & -1 \\
1 & -1 & -1 & 0 & 0 \\
\left(c_{11}^{c}+c_{12}^{c}\right) & -\left(c_{11}^{s}+c_{12}^{s}\right) & \left(c_{11}^{s}-c_{12}^{s}\right) & c_{13}^{c} & -c_{13}^{s} \\
\eta 2 c_{13}^{c} & (1-\eta) 2 c_{13}^{s} & 0 & \eta c_{33}^{c} & (1-\eta) c_{33}^{s} \\
0 & \left(c_{11}^{s}+c_{12}^{s}\right) & -\eta\left(c_{11}^{s}-c_{12}^{s}\right) & 0 & c_{13}^{s}
\end{array}\right) \\
\times\left(\begin{array}{c}
A^{c} \\
A^{s} \\
B^{s} \\
C^{c} \\
C^{s}
\end{array}\right)=\left(\begin{array}{c}
f_{\|} \\
f_{\perp} \\
0 \\
0 \\
0
\end{array}\right) .
\end{gathered}
$$

A direct numerical calculation is possible, however it is interesting to write the boundary conditions using a transfer matrix method, which can be generalized to multishell NWs [9]: then, we have to solve a system of two linear equations, instead of 5 for a core-shell NW and $(3 n+2)$ for a NW with $(n-1)$ shells.

\subsubsection{Transfer matrix}

We thus consider a multishell NW made of a core of radius $r_{0}$, and several layers of radius $r_{i}$ and lattice mismatch $f_{\| i}$ and $f_{\perp i}$ with respect to the core material, with a uniform stiffness tensor over the whole NW. The radius of the last layer, $i=s$, is the NW radius. The relative cross-section area of each layer is $\eta_{i}=\left(r_{i}^{2}-r_{i-1}^{2}\right) /\left(r_{s}^{2}\right)$.

Within each material, we define a matrix $\mathbf{M}(\rho)$ relating the relevant components of displacement and stress to the $A, B, C$ parameters, with $\rho=r / r_{0}$ :

$$
\left(\begin{array}{c}
\frac{u_{z}}{z} \\
\frac{u_{r}}{r} \\
\sigma_{r r}
\end{array}\right)=\mathbf{M}(\rho)\left(\begin{array}{l}
C \\
A \\
B
\end{array}\right)
$$

with

$$
\begin{aligned}
\mathbf{M}(\rho) & =\left(\begin{array}{ccc}
1 & 0 & 0 \\
0 & 1 & \rho^{-2} \\
c_{13} & \left(c_{11}+c_{12}\right) & -\left(c_{11}-c_{12}\right) \rho^{-2}
\end{array}\right) \\
& =\mathbf{M}(1)\left(\begin{array}{ccc}
1 & 0 & 0 \\
0 & 1 & 0 \\
0 & 0 & \rho^{-2}
\end{array}\right)
\end{aligned}
$$


and

$$
\mathbf{M}^{-1}(\rho)=\frac{1}{2 c_{11}}\left(\begin{array}{ccc}
2 c_{11} & 0 & 0 \\
-c_{13} & \left(c_{11}-c_{12}\right) & 1 \\
c_{13} \rho^{2} & \left(c_{11}+c_{12}\right) \rho^{2} & -\rho^{2}
\end{array}\right) .
$$

In the general case, the values of the stiffness constants are specific to the material which makes the layer $i$, and accordingly there is a matrix $\mathbf{M}_{i}$ appropriate to each material.

The boundary condition at the interface between layers $i$ and $(i+1)$, at $\rho=\rho_{i}$, is:

$\mathbf{M}_{i+1}\left(\rho_{i}\right)\left(\begin{array}{c}C_{i+1} \\ A_{i+1} \\ B_{i+1}\end{array}\right)=\mathbf{M}_{i}\left(\rho_{i}\right)\left(\begin{array}{c}C_{i} \\ A_{i} \\ B_{i}\end{array}\right)-\left(\begin{array}{c}f_{\|(i+1)} \\ f_{\perp(i+1)} \\ 0\end{array}\right)+\left(\begin{array}{c}f_{\| i} \\ f_{\perp} i \\ 0\end{array}\right)$.

This condition can be re-written:

$$
\begin{aligned}
\left(\begin{array}{c}
C_{i+1} \\
A_{i+1} \\
B_{i+1}
\end{array}\right)= & \mathbf{M}_{i+1}^{-1}\left(\rho_{i}\right) \mathbf{M}_{i}\left(\rho_{i}\right)\left(\begin{array}{c}
C_{i} \\
A_{i} \\
B_{i}
\end{array}\right) \\
& -\mathbf{M}_{i+1}^{-1}\left(\rho_{i}\right)\left[\left(\begin{array}{c}
f_{\|(i+1)} \\
f_{\perp(i+1)} \\
0
\end{array}\right)-\left(\begin{array}{c}
f_{\| i} \\
f_{\perp} i \\
0
\end{array}\right)\right] .
\end{aligned}
$$

Equation (12) establishes a relation of recurrence from the parameters on the inner side of the interface, to those on the outer side. Repeating equation (12) from shell to shell, we obtain the set of parameters $\left(C_{i}, A_{i}, B_{i}\right)$ as a function of those of the core, $\left(C_{0}, A_{0}\right.$, with $\left.B_{0}=0\right)$. The two core parameters are finally determined by the two boundary conditions on the stress at the sidewall and along $z$.

The first boundary conditions, $\sigma_{r r}=0$ at the sidewall, is written using the projection ${ }^{t} \mathbf{P}_{r}=\left(\begin{array}{lll}0 & 0 & 1\end{array}\right)$,

$$
{ }^{t} \mathbf{P}_{r}\left(\begin{array}{c}
\frac{u_{z}}{z} \\
\frac{u_{r}}{r} \\
\sigma_{r r}
\end{array}\right)=0
$$

at the surface $\left(r=r_{s}\right)$, hence

$$
{ }^{t} \mathbf{P}_{r} \mathbf{M}_{s}\left(\rho_{s}\right)\left(\begin{array}{l}
C_{s} \\
A_{s} \\
B_{s}
\end{array}\right)=0
$$

The last condition, on $\sigma_{z z}$ integrated over the NW crosssection, is:

$$
\sum_{i=0}^{s} \eta_{i}{ }^{t} \mathbf{P}_{i z}\left(\begin{array}{c}
C_{i} \\
A_{i} \\
B_{i}
\end{array}\right)=0
$$

with ${ }^{t} \mathbf{P}_{i z}=\left(\begin{array}{lll}c_{33} & 2 c_{13} & 0\end{array}\right)$ written with the values of stiffness constants appropriate to the material in layer $i$. Combining equations (12)-(14) we obtain a set of two linear equations for $A_{0}$ and $C_{0}$.

\subsubsection{The case of uniform Poisson ratios}

If we assume a common value of the Poisson ratios in the different materials, as in reference [12] (i.e., if $c_{i j k l}^{i} / c_{i j k l}^{0}$ takes a single value $\chi_{i}$ ), several explicit expressions are obtained.

With this assumption, the continuity of $\sigma_{r r}$ at the interface at $r_{i}$ (last line of Eq. (11), multiplied by $\rho_{i}^{2}$ ) is:

$$
\begin{gathered}
\chi_{i}\left[c_{13} C_{i} \rho_{i}^{2}+\left(c_{11}+c_{12}\right) A_{i} \rho_{i}^{2}-\left(c_{11}-c_{12}\right) B_{i}\right] \\
=\chi_{i+1}\left[c_{13} C_{i+1} \rho_{i}^{2}+\left(c_{11}+c_{12}\right) A_{i+1} \rho_{i}^{2}\right. \\
\left.-\left(c_{11}-c_{12}\right) B_{i+1}\right] .
\end{gathered}
$$

Adding these equations for all interfaces, including the surface for which the right-hand member is zero, and using $\eta_{i}=\left(\rho_{i}^{2}-\rho_{i-1}^{2}\right) / \rho_{s}^{2}$, we obtain:

$$
c_{13} \sum_{i=0}^{s} C_{i} \chi_{i} \eta_{i}+\left(c_{11}+c_{12}\right) \sum_{i=0}^{s} A_{i} \chi_{i} \eta_{i}=0 .
$$

The second condition is that the integral of $\sigma_{z z}$ over the NW cross-section vanishes:

$$
c_{33} \sum_{i=0}^{s} C_{i} \chi_{i} \eta_{i}+2 c_{13} \sum_{i=0}^{s} A_{i} \chi_{i} \eta_{i}=0 .
$$

Hence the two sums must vanish independently:

$$
\begin{aligned}
& \sum_{i=0}^{s} C_{i} \chi_{i} \eta_{i}=0 \\
& \sum_{i=0}^{s} A_{i} \chi_{i} \eta_{i}=0 .
\end{aligned}
$$

Another simple result is obtained for the strain along the axis. The first line of equation (11) or (12), $C_{i+1}=C_{i}-$ $f_{\| i+1}+f_{\| i}$, results in $C_{i}=C_{0}-f_{\| i}$ and finally, using the first sum rule $\sum_{i=0}^{s} C_{i} \chi_{i} \eta_{i}=0$,

$$
C_{i}=\sum_{j=0}^{s} \chi_{j} \eta_{j} f_{\| j}-f_{\| i}
$$

The recurrence on the in-plane strain is not as simple. Indeed the transfer matrix in equation (12) is:

$$
\begin{aligned}
& \mathbf{M}_{i+1}^{-1}\left(\rho_{i}\right) \mathbf{M}_{i}\left(\rho_{i}\right)=\mathbf{1} \\
& +\frac{\chi_{i}-\chi_{i+1}}{\chi_{i+1}} \frac{1}{2 c_{11}}\left(\begin{array}{ccc}
0 & 0 & 0 \\
c_{13} & \left(c_{11}+c_{12}\right) & -\left(c_{11}-c_{12}\right) \\
-c_{13} \rho^{2} & -\left(c_{11}+c_{12}\right) \rho^{2} & \left(c_{11}-c_{12}\right) \rho^{2}
\end{array}\right),
\end{aligned}
$$

which shows that if $\chi_{i+1} \neq \chi_{i}$, only the recurrence on $C_{i}$ is simple.

It is worth however to write the result for the simple core-shell NW. Simplifying the notation, with $\eta=\eta_{0}$, 
D. Ferrand and J. Cibert: Strain in crystalline core-shell nanowires

$\left.\chi=\chi_{s}, \chi_{0}=1, f=f_{s}\right)$,

$$
\begin{aligned}
\varepsilon_{z z}^{c} & =\frac{(1-\eta) \chi}{\eta+(1-\eta) \chi} f_{\|} \\
\varepsilon_{z z}^{s} & =\frac{-\eta}{\eta+(1-\eta) \chi} f_{\|} \\
\frac{\varepsilon_{\theta \theta}^{c}-\varepsilon_{r r}^{c}}{2} & =0 \\
\frac{\varepsilon_{\theta \theta}^{s}-\varepsilon_{r r}^{s}}{2} & =B_{s} \frac{r_{c}^{2}}{r^{2}} \\
\frac{\varepsilon_{\theta \theta}^{c}+\varepsilon_{r r}^{c}}{2} & =\frac{(1-\eta) \chi}{\eta+(1-\eta) \chi}\left(f_{\perp}+B_{s}\right) \\
\frac{\varepsilon_{\theta \theta}^{s}+\varepsilon_{r r}^{s}}{2} & =\frac{-\eta}{\eta+(1-\eta) \chi}\left(f_{\perp}+B_{s}\right),
\end{aligned}
$$

where

$$
\begin{aligned}
B_{s} & =-\frac{\left(c_{11}+c_{12}\right) f_{\perp}+c_{13} f_{\|}}{\left(c_{11}-c_{12}\right)[\eta+(1-\eta) \chi]+\left(c_{11}+c_{12}\right)} \\
f_{\perp}+B_{s} & =\frac{\left(c_{11}-c_{12}\right)[\eta+(1-\eta) \chi] f_{\perp}-c_{13} f_{\|}}{\left(c_{11}-c_{12}\right)[\eta+(1-\eta) \chi]+\left(c_{11}+c_{12}\right)} .
\end{aligned}
$$

\subsubsection{The case of a uniform stiffness tensor}

If all materials have the same values of stiffness constants (all $\chi_{i}=1$ ), all $\mathbf{M}_{i}$ matrices are identical. The recurrence relation (Eq. (12)) is simply:

$$
\begin{aligned}
\left(\begin{array}{l}
C_{i+1} \\
A_{i+1} \\
B_{i+1}
\end{array}\right)= & \left(\begin{array}{c}
C_{i} \\
A_{i} \\
B_{i}
\end{array}\right) \\
& -\mathbf{M}^{-1}\left(\rho_{i}\right)\left[\left(\begin{array}{c}
f_{\|(i+1)} \\
f_{\perp(i+1)} \\
0
\end{array}\right)-\left(\begin{array}{c}
f_{\| i} \\
f_{\perp} i \\
0
\end{array}\right)\right],
\end{aligned}
$$

or

$$
\begin{aligned}
C_{i+1}= & C_{i}+f_{\| i}-f_{\|(i+1)} \\
A_{i+1}= & A_{i}+\frac{c_{11}-c_{12}}{2 c_{11}}\left(f_{\perp i}-f_{\perp(i+1)}\right) \\
& -\frac{c_{13}}{2 c_{11}}\left(f_{\| i}-f_{\|(i+1)}\right) \\
B_{i+1}= & B_{i}+\frac{c_{11}+c_{12}}{2 c_{11}}\left(f_{\perp i}-f_{\perp(i+1)}\right) \rho_{i}^{2} \\
& +\frac{c_{13}}{2 c_{11}}\left(f_{\| i}-f_{\|(i+1)}\right) \rho_{i}^{2},
\end{aligned}
$$

so that

$$
\begin{aligned}
C_{i}= & C_{0}-f_{\| i} \\
A_{i}= & A_{0}-\frac{c_{11}-c_{12}}{2 c_{11}} f_{\perp i}+\frac{c_{13}}{2 c_{11}} f_{\| i} \\
B_{i}= & \frac{c_{11}+c_{12}}{2 c_{11}}\left[\sum_{j=0}^{i-1} \eta_{j} f_{\perp j} \rho_{s}^{2}-f_{\perp i} \rho_{i-1}^{2}\right] \\
& +\frac{c_{13}}{2 c_{11}}\left[\sum_{j=0}^{i-1} \eta_{j} f_{\| j} \rho_{s}^{2}-f_{\| i} \rho_{i-1}^{2}\right],
\end{aligned}
$$

and finally, using equation (16):

$$
\begin{aligned}
C_{i}= & \sum_{j=0}^{s} \eta_{j} f_{\| j}-f_{\| i} \\
A_{i}= & \frac{\left(c_{11}-c_{12}\right)}{2 c_{11}}\left[\sum_{j=0}^{s} \eta_{j} f_{\perp j}-f_{\perp i}\right] \\
& -\frac{c_{13}}{2 c_{11}}\left[\sum_{j=0}^{s} \eta_{j} f_{\| j}-f_{\| i}\right] .
\end{aligned}
$$

The strain configuration is thus:

$$
\begin{aligned}
\left(\varepsilon_{z z}\right)_{i}= & \sum_{j=0}^{s} \eta_{j} f_{\| j}-f_{\| i} \\
\left(\frac{\varepsilon_{\theta \theta}-\varepsilon_{r r}}{2}\right)_{i}= & \frac{c_{11}+c_{12}}{2 c_{11}} \\
& \times\left[\sum_{j=0}^{i-1} \eta_{j} f_{\perp j}\left(\frac{r_{s}}{r}\right)^{2}-f_{\perp i}\left(\frac{r_{i-1}}{r}\right)^{2}\right] \\
& +\frac{c_{13}}{2 c_{11}}\left[\sum_{j=0}^{i-1} \eta_{j} f_{\| j}\left(\frac{r_{s}}{r}\right)^{2}-f_{\| i}\left(\frac{r_{i-1}}{r}\right)^{2}\right] \\
\left(\frac{\varepsilon_{\theta \theta}+\varepsilon_{r r}}{2}\right)_{i}= & \frac{\left(c_{11}-c_{12}\right)}{2 c_{11}}\left[\sum_{j=0}^{s} \eta_{j} f_{\perp j}-f_{\perp i}\right] \\
& -\frac{c_{13}}{2 c_{11}}\left[\sum_{j=0}^{s} \eta_{j} f_{\| j}-f_{\| i}\right] .
\end{aligned}
$$

It can be applied to a multishell structure such as in reference [15].

In the case of the simple core-shell NW, we recover the usual expressions, equation (5):

$$
\begin{aligned}
\varepsilon_{z z}^{c} & =(1-\eta) f_{\|} \\
\varepsilon_{z z}^{s} & =-\eta f_{\|} \\
\frac{\varepsilon_{\theta \theta}^{s}-\varepsilon_{r r}^{s}}{2} & =B s \frac{r_{c}^{2}}{r^{2}} \\
\varepsilon_{\theta \theta}^{c}=\varepsilon_{r r}^{c} & =(1-\eta)\left(f_{\perp}+B_{s}\right) \\
\frac{\varepsilon_{\theta \theta}^{s}+\varepsilon_{r r}^{s}}{2} & =-\eta\left(f_{\perp}+B_{s}\right),
\end{aligned}
$$

where

$$
\begin{array}{r}
B_{s}=-\frac{\left(c_{11}+c_{12}\right) f_{\perp}+c_{13} f_{\|}}{2 c_{11}} \\
f_{\perp}+B_{s}=\frac{\left(c_{11}-c_{12}\right) f_{\perp}-c_{13} f_{\|}}{2 c_{11}} .
\end{array}
$$

A comparison between the expressions for $\varepsilon_{z z}$ in equations (18)-(19) and equations (25)-(26) illustrates the effect of a different hardness of the two materials: in the 
sharing of lattice mismatch, the weight is defined by the area ratio multiplied by the hardness ratio. In particular, for a thin layer $(\eta \approx 1)$, the strain in the core is multiplied by $\chi$, while for a thick layer $(\eta \ll 1)$, the strain in the shell is divided by $\chi$.

Note also that equation (24) can be used to describe a continuous distribution in a NW, just by replacing the discrete sums by integrals:

$$
\begin{aligned}
C_{0}= & \sum_{j=0}^{s} \eta_{j} f_{\| j} \rightarrow \int f_{\|}(r) \frac{2 r d r}{r_{s}^{2}} \\
A_{0}= & \frac{\left(c_{11}-c_{12}\right)}{2 c_{11}} \sum_{j=0}^{s} \eta_{j} f_{\perp j}-\frac{c_{13}}{2 c_{11}} \sum_{j=0}^{s} \eta_{j} f_{\| j} \\
& \rightarrow \frac{\left(c_{11}-c_{12}\right)}{2 c_{11}} \int f_{\perp}(r) \frac{2 r d r}{r_{s}^{2}}-\frac{c_{13}}{2 c_{11}} \int f_{\|}(r) \frac{2 r d r}{r_{s}^{2}} .
\end{aligned}
$$

An interesting consequence is that the strain in the core of a core-shell NW (for instance, GaAs- $\mathrm{Ga}_{1-x} \mathrm{Al}_{x} \mathrm{As}$ ) or in a multishell NW, is determined by the composition integrated over the shell(s), and not by the exact distribution within the shell(s). The analogy with the Gauss theorem of electrostatics is not fortuitous and it has been discussed in reference [16] for a quantum dot buried in an isotropic material. However it applies only in special cases where the (vectorial) Lamé-Clapeyron-Navier equation can be mapped onto the scalar Poisson equation, with the local mismatch defining the equivalent of the electric charge.

\subsubsection{Summary and electronic properties}

To sum up, the strain configuration in a core-shell NW grown along the hexagonal direction of wurtzite crystal is transversely isotropic. It is given by equations (25) and (26) if the two materials have the same hardness, and equations (18) and (19) for a hardness ratio $\chi \neq 1$. An explicit expression, equation (24), also exists for a multishell NW if the stiffness constants are identical over the NW.

The potential configuration for the bottom of the conduction band and the top of the valence band near the center of the Brillouin zone is obtained from these expressions using the Bir-Pikus phenomenological coupling [11]. In the core, the non-vanishing strain components are $\varepsilon_{z z}$ and $\frac{1}{2}\left(\varepsilon_{r r}+\varepsilon_{\theta \theta}\right)$ so that the Bir-Pikus Hamiltonian [11] has only diagonal elements in the usual basis quantized along the $c$-axis. Note however that the resulting matrix elements may be of the same order as the other terms describing the top of the valence band and the excitons (spinorbit coupling, crystal field splitting and exchange terms). In the shell $(\mathrm{s})$, the in-plane shear strain $\frac{1}{2}\left(\varepsilon_{r r}-\varepsilon_{\theta \theta}\right)$ introduces non-diagonal terms, which mix the valence band states initially quantized along the $c$-axis. Actually, this term may give the main contribution to the hole potential in the shell. Interestingly, it splits the hole multiplet in such a way that one type of holes is confined in the vicinity of the interface, far from the sidewall.

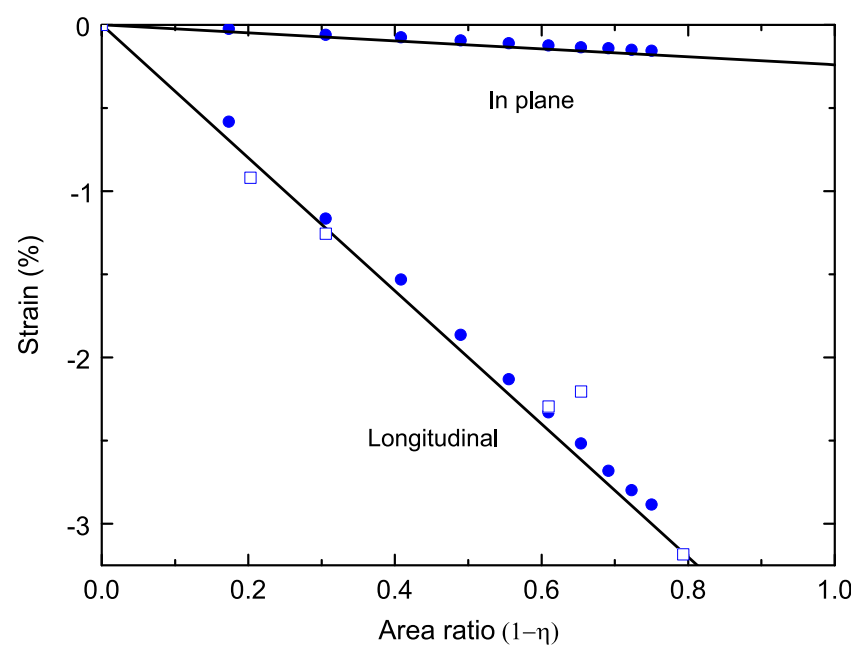

Fig. 2. Strain in the core of a GaN-AlN NW, as a function of the area ratio. Solid symbols, microscopic calculation, open symbols, electron microscopy data, both from reference [17]; lines, present calculation.

The piezoelectric effect is described by an axial polarization, determined by the two strain components $\varepsilon_{z z}$ and $\frac{1}{2}\left(\varepsilon_{r r}+\varepsilon_{\theta \theta}\right)$. There is no coupling to the in-plane shear strain.

The present study also confirms that GaN-InN multiquantum-well NWs [15] should indeed feature no built-in piezoelectric field perpendicular to the QWs, but an inplane shear-strain different from well to well.

\subsection{Application to real systems}

\subsubsection{GaN-AIN nanowires}

The strain in the core of single GaN-AlN core-shell NWs grown by plasma-assisted molecular beam epitaxy was measured by resonant X-ray diffraction, Raman spectroscopy and high resolution transmission electron microscopy [17]: for unrelaxed NWs, it favorably compares to the results of a microscopic calculation using the valenceforce-field model, and to a macroscopic calculation assuming uniform strain along the $c$-axis and vanishing strain in the plane. Complementary results are given in reference [18].

The stiffness constants of GaN and AlN [19-21] are quite similar, hence we take $\chi=1$. The lattice mismatch is slightly anisotropic, $f_{\perp}=-2.5 \%$ and $f_{\|}=-4.0 \%$. The present calculation predicts a uniform strain in the core, $\varepsilon_{z z}^{c}=f_{\perp}(1-\eta)$ along the NW axis and $\frac{1}{2}\left(\varepsilon_{\theta \theta}^{c}+\varepsilon_{r r}^{c}\right)=$ $-(1-\eta) \times 0.25 \%$ in the plane. The agreement with the results of reference [17] is excellent, see Figure 2.

Note that the small value of the in-plane strain is due to a compensation between the Poisson effect of the longitudinal mismatch $f_{\|}$and the direct effect of the in-plane mismatch $f_{\perp}$, see $f_{\perp}+B_{s}$ in equation (26). 
D. Ferrand and J. Cibert: Strain in crystalline core-shell nanowires

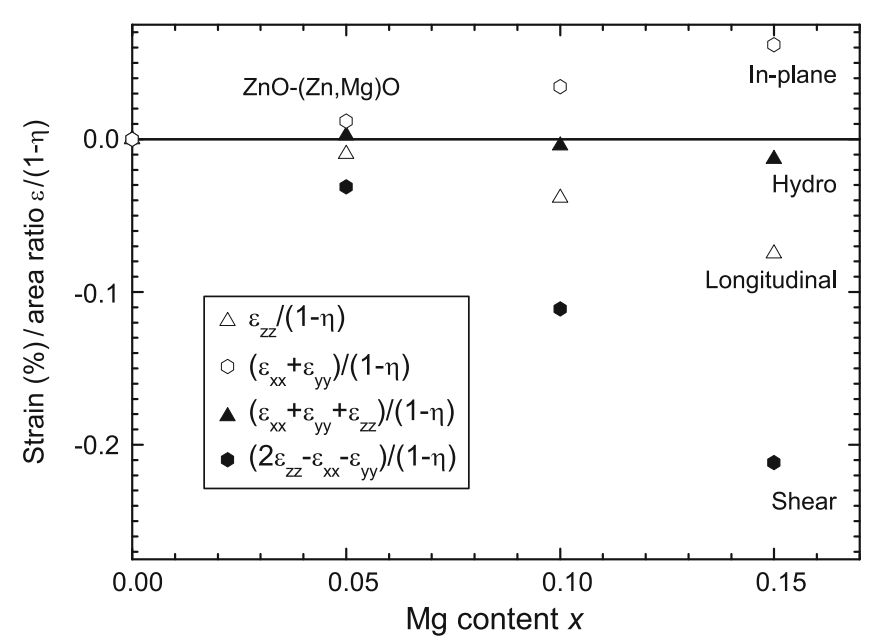

Fig. 3. Strain in the core of a $\mathrm{ZnO}-(\mathrm{Zn}, \mathrm{Mg}) \mathrm{O}$ NW, divided by the shell/NW area ratio $(1-\eta)$, as a function of the $\mathrm{Mg}$ content $x$. The values of the lattice mismatch along the NW axis and perpendicular to it are the experimental values of reference [17] and are opposite in sign. Open symbols, longitudinal and inplane strain. Full symbols, shear strain and volume change.

\subsection{2 $\mathrm{ZnO}$ nanowires}

$\mathrm{ZnO}$ is such that $c_{11}-c_{12}<c_{13}$ [22]: for an isotropic lattice mismatch, the Poisson effect prevails in the in-plane strain. $\mathrm{ZnO}$ cores are often associated to a strongly mismatched shell and in this case the structure is no more coherent. A moderate mismatch exists in $\mathrm{ZnO}-(\mathrm{Zn}, \mathrm{Mg}) \mathrm{O}$. According to a synchrotron X-ray study of polycrystalline wurtzite $(\mathrm{Zn}, \mathrm{Mg}) \mathrm{O}$ [23], it is strongly anisotropic, with $f_{\perp}$ and $f_{\|}$opposite in sign: this is attributed to a change in the ionicity. As a result (Fig. 3), the core experiences a significant shear strain with a 10 times smaller volume change. In other words, the $c / a$ ratio, which represents the deviation from "ideal" wurtzite, is changed at almost constant volume. Note that a non-linear character of the piezoelectric effect has been measured in CdTe [24] and predicted for other semiconductors as well [25]. As it is attributed to a dependence of the piezoelectric coefficient on the hydrostatic strain, this non-linear character should not show up in a $\mathrm{ZnO}-(\mathrm{Zn}, \mathrm{Mg}) \mathrm{O} \mathrm{NW}$.

\section{Cubic semiconductors along $\langle 111\rangle$}

We now consider NWs of semiconductors with the zincblende or diamond structure, grown along a trigonal axis. The (111) plane is known to be isotropic with respect to some mechanical properties, so that the cylindrical approximation is quite natural for such NWs. We use it first, and compare its results to data known for real systems. However the shear strain present in the shell gives rise to warping, with a three-fold symmetry, which is calculated analytically in Section 4.3.2.

\subsection{Cylindrical approximation}

\subsubsection{Calculation}

If the parameter $c$ is not zero, the stiffness tensor must be calculated in the relevant axes. It can be done on the $c_{i j k l}$ tensor, or directly in the Voigt notation using the rotation rules described in reference [13]. We take the basis defined by the three vectors $x=[1 \overline{1} 0], y=[11 \overline{2}], z=$ [111], identical to that in reference [6] but different from reference [26]. Then [8] the stiffness matrix is:

$$
\left(\begin{array}{cccccc}
\tilde{c}_{11} & \tilde{c}_{12} & \tilde{c}_{13} & \tilde{c}_{14} & 0 & 0 \\
\tilde{c}_{12} & \tilde{c}_{11} & \tilde{c}_{13} & -\tilde{c}_{14} & 0 & 0 \\
\tilde{c}_{13} & \tilde{c}_{13} & \tilde{c}_{33} & 0 & 0 & 0 \\
\tilde{c}_{14} & -\tilde{c}_{14} & 0 & \tilde{c}_{44} & 0 & 0 \\
0 & 0 & 0 & 0 & \tilde{c}_{44} & \tilde{c}_{14} \\
0 & 0 & 0 & 0 & \tilde{c}_{14} & \frac{\tilde{c}_{11}-\tilde{c}_{12}}{2}
\end{array}\right) .
$$

The six components are not independent since they can be expressed using the three coefficients $c_{11}, c_{12}$ and $c_{44}$ relevant for the cubic symmetry [6]:

$$
\begin{aligned}
& \tilde{c}_{11}=c_{11}-\frac{1}{2} c=\frac{1}{2} c_{11}+\frac{1}{2} c_{12}+c_{44} \\
& \tilde{c}_{33}=c_{11}-\frac{2}{3} c=\frac{1}{3} c_{11}+\frac{2}{3} c_{12}+\frac{4}{3} c_{44} \\
& \tilde{c}_{12}=c_{12}+\frac{1}{6} c=\frac{1}{6} c_{11}+\frac{5}{6} c_{12}-\frac{2}{6} c_{44} \\
& \tilde{c}_{13}=c_{12}+\frac{1}{3} c=\frac{1}{3} c_{11}+\frac{2}{3} c_{12}-\frac{2}{3} c_{44} \\
& \tilde{c}_{44}=c_{44}+\frac{1}{3} c=\frac{1}{3} c_{11}-\frac{1}{3} c_{12}+\frac{1}{3} c_{44} \\
& \tilde{c}_{14}=\frac{1}{3 \sqrt{2}} c=\frac{1}{3 \sqrt{2}}\left(c_{11}-c_{12}-2 c_{44}\right) .
\end{aligned}
$$

The stiffness tensor reflects the threefold symmetry of the trigonal axis: it is quite similar to that of the wurtzite structure along the $c$-axis. However there is a set of additional terms, $\tilde{c}_{14}$. To better understand these terms, we can write the stiffness matrix in the $\mathbf{e}_{r}, \mathbf{e}_{\theta}, \mathbf{e}_{z}$ axes, rotated with respect to the previous one by an angle $\theta$ around the $\langle 111\rangle$ (or $\mathbf{e}_{z}$ ) axis:

$$
\begin{aligned}
& \left(\begin{array}{cccccc}
\tilde{c}_{11} & \tilde{c}_{12} & \tilde{c}_{13} & 0 & 0 & 0 \\
\tilde{c}_{12} & \tilde{c}_{11} & \tilde{c}_{13} & 0 & 0 & 0 \\
\tilde{c}_{13} & \tilde{c}_{13} & \tilde{c}_{33} & 0 & 0 & 0 \\
0 & 0 & 0 & \tilde{c}_{44} & 0 & 0 \\
0 & 0 & 0 & 0 & \tilde{c}_{44} & 0 \\
0 & 0 & 0 & 0 & 0 & \frac{\tilde{c}_{11}-\tilde{c}_{12}}{2}
\end{array}\right)+ \\
& \tilde{c}_{14}\left(\begin{array}{cccccc}
0 & 0 & 0 & \cos 3 \theta & \sin 3 \theta & 0 \\
0 & 0 & 0 & -\cos 3 \theta & -\sin 3 \theta & 0 \\
0 & 0 & 0 & 0 & 0 & 0 \\
\cos 3 \theta & -\cos 3 \theta & 0 & 0 & 0 & \sin 3 \theta \\
\sin 3 \theta & -\sin 3 \theta & 0 & 0 & 0 & \cos 3 \theta \\
0 & 0 & 0 & \sin 3 \theta & \cos 3 \theta & 0
\end{array}\right) .
\end{aligned}
$$

The trigonal symmetry of the $\tilde{c}_{14}$ terms is clear, as noted in reference [27]. Note that these contributions average to 
zero over a complete $2 \pi$-turn. Moreover, they are quite small: for instance in GaAs, $\tilde{c}_{14} /\left(\tilde{c}_{11}+2 \tilde{c}_{12}\right)=-0.05$.

The complete Lamé-Clapeyron-Navier equation in the $x, y, z$ basis, equation (1), is written in Appendix A. Omitting terms excluded by the invariance by translation, we obtain:

$$
\begin{gathered}
\frac{\tilde{c}_{11}-\tilde{c}_{12}}{2}\left(\frac{\partial^{2}}{\partial y^{2}}+\frac{\partial^{2}}{\partial x^{2}}\right) u_{x} \\
+\frac{\tilde{c}_{11}+\tilde{c}_{12}}{2} \frac{\partial}{\partial x}\left(\frac{\partial u_{x}}{\partial x}+\frac{\partial u_{y}}{\partial y}\right) \\
\quad+2 \tilde{c}_{14}\left(\frac{\partial^{2} u_{x}}{\partial y \partial z}+\frac{\partial^{2} u_{y}}{\partial z \partial x}+\frac{\partial^{2} u_{z}}{\partial x \partial y}\right)=0 \\
\frac{\tilde{c}_{11}-\tilde{c}_{12}}{2}\left(\frac{\partial^{2}}{\partial y^{2}}+\frac{\partial^{2}}{\partial x^{2}}\right) u_{y} \\
\quad+\frac{\tilde{c}_{11}}{2} \tilde{c}_{12} \frac{\partial}{\partial y}\left(\frac{\partial u_{x}}{\partial x}+\frac{\partial u_{y}}{\partial y}\right) \\
\quad+\tilde{c}_{14}\left(2 \frac{\partial^{2} u_{x}}{\partial z \partial x}+\frac{\partial^{2} u_{z}}{\partial x^{2}}-2 \frac{\partial^{2} u_{y}}{\partial y \partial z}-\frac{\partial^{2} u_{z}}{\partial y^{2}}\right)=0 \\
\quad\left(\frac{\partial^{2}}{\partial y^{2}}+\frac{\partial^{2}}{\partial x^{2}}\right) u_{z} \\
\quad+\tilde{c}_{14}\left(2 \frac{\partial^{2} u_{x}}{\partial x \partial y}+\frac{\partial^{2} u_{y}}{\partial x^{2}}-\frac{\partial^{2} u_{y}}{\partial y^{2}}\right)=0 .
\end{gathered}
$$

The effect of the $\tilde{c}_{14}$ terms will be described in Section 4.3.2. Ignoring these terms for a while, the equation is the same as in the wurtzite case. Then the solution is obtained by replacing the $c_{i j}$ in equations (18) and (19) by the $\tilde{c}_{i j}$ and their expression (Eq. 29). The result is identical to equation (18), but with $f_{\|}=f_{\perp}=f$, and:

$B_{s}=$

$-\frac{3\left(c_{11}+2 c_{12}\right)}{\left(c_{11}-c_{12}+4 c_{44}\right)[\eta+(1-\eta) \chi]+\left(2 c_{11}+4 c_{12}+2 c_{44}\right)} f$

$f+B_{s}=$

$\frac{\left(c_{11}-c_{12}+4 c_{44}\right)[\eta+(1-\eta) \chi]-\left(c_{11}+2 c_{12}-2 c_{44}\right)}{\left(c_{11}-c_{12}+4 c_{44}\right)[\eta+(1-\eta) \chi]+\left(2 c_{11}+4 c_{12}+2 c_{44}\right)} f$.

If the stiffness constants are identical in the two materials we recover the same expression as above (Eq. (5)), with:

$$
\begin{aligned}
B_{s} & =-f \frac{c_{11}+2 c_{12}}{c_{11}+c_{12}+2 c_{44}} \\
\left(f+B_{s}\right) & =f \frac{-c_{12}+2 c_{44}}{c_{11}+c_{12}+2 c_{44}} .
\end{aligned}
$$

In the core, the strain corresponds to a uniform hydrostatic strain $\varepsilon_{\text {hydro }}=\varepsilon_{z z}+\varepsilon_{r r}+\varepsilon_{\theta \theta}$ and a uniform trigonal shear strain $\varepsilon_{\text {shear }}=2 \varepsilon_{z z}-\varepsilon_{r r}-\varepsilon_{\theta \theta}$. It should be kept in mind that the axis used in these expression are $x=\left[\begin{array}{ll}1 & 1\end{array}\right]$,

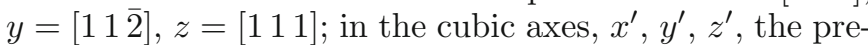
vious results means, for the core, $\varepsilon_{x^{\prime} x^{\prime}}=\varepsilon_{y^{\prime} y^{\prime}}=\varepsilon_{z^{\prime} z^{\prime}}=$ $\frac{1}{3} \varepsilon_{\text {hydro }}$ and $\varepsilon_{x^{\prime} y^{\prime}}=\varepsilon_{y^{\prime} z^{\prime}}=\varepsilon_{z^{\prime} x^{\prime}}=\frac{1}{6} \varepsilon_{\text {shear }}$

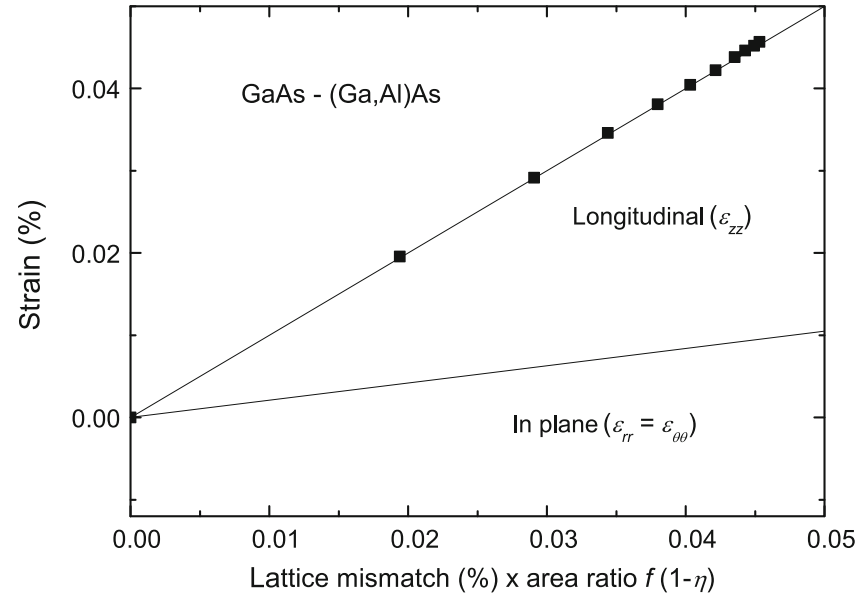

Fig. 4. Strain in a GaAs core, as a function of area ratio $(1-\eta)$ times the lattice mismatch $f$. Symbols are the numerical calculation of reference [28], lines are the present calculation.

\subsubsection{Excitons}

Finally, we consider the exciton energy in the core of a core-shell NW, in the absence of confinement effects. In a strained semiconductor, it is expected at $E_{X}=E_{X}^{0}-$ $\left(a^{\prime}+a\right) \varepsilon_{\text {hydro }} \pm \frac{1}{2} b \varepsilon_{\text {shear }}$ (for a tetragonal shear strain) or $E_{X}=E_{X}^{0}-\left(a^{\prime}+a\right) \varepsilon_{\text {hydro }} \pm \frac{1}{2}(d / \sqrt{3}) \varepsilon_{\text {shear }}$ (for a trigonal shear strain). The coefficient $a^{\prime}$ describes the coupling of conduction electrons to strain, and $a, b, d$ describe the coupling of holes (Bir-Pikus Hamiltonian [11]). Using equation (33) we obtain:

$$
\begin{array}{r}
E_{X}=E_{X}^{0}-\left(a^{\prime}+a\right) \frac{\left(c_{11}-c_{12}+6 c_{44}\right)}{c_{11}+c_{12}+2 c_{44}}(1-\eta) f \\
\pm \frac{d}{\sqrt{3}} \frac{\left(c_{11}+2 c_{12}\right)}{c_{11}+c_{12}+2 c_{44}}(1-\eta) f .
\end{array}
$$

The sign + is for the exciton formed with the light hole (moment $\pm \frac{1}{2}$ along the NW axis), the sign - for the heavy hole $\left( \pm \frac{3}{2}\right)$ exciton. A more complete analysis is given at the end of Section 4.3.2.

\subsection{Application to real systems}

\subsubsection{GaAs-based nanowires}

The calculation for a $\mathrm{GaAs}-\mathrm{Ga}_{0.65} \mathrm{Al}_{0.35} \mathrm{As} \mathrm{NW}$ with hexagonal cross-section, using the valence force field model, Figure 3c of reference [28], fully agrees (Fig. 4) with the present value $\varepsilon_{z z}^{c}=(1-\eta) f$. The in-plane strain is "four times smaller" [28], which also agrees with the ratio 0.22 obtained in the present calculation using the stiffness constants of $(\mathrm{Ga}, \mathrm{Al}) \mathrm{As}[29]$, with $\chi=1$.

In GaAs-GaP NWs, the lattice mismatch is $3.6 \%$, and the stiffness constants differ by a factor $\chi \approx 1.10$ to 1.17 [30]. NWs with either a circular or a hexagonal crosssections have been modeled by Grönqvist et al. [6] using both the valence force field model and a finite element treatment of the continuum elasticity theory. Other 


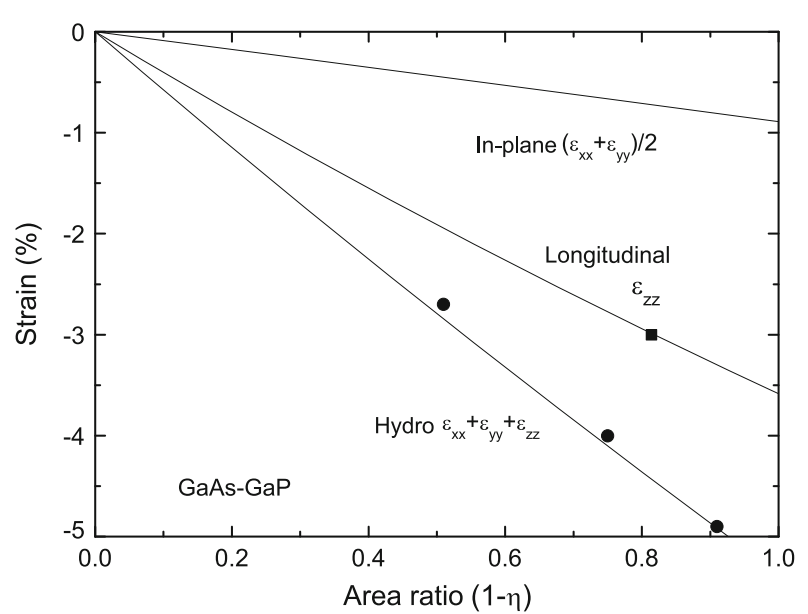

Fig. 5. Strain in a GaAs core with a $\mathrm{GaP}$ shell, as a function of area ratio $(1-\eta)$. Symbols are the numerical calculation of reference [6] (square) and reference [31] (circles), lines are the present calculation.

core-shell configurations with hexagonal cross-sections are described in reference [31]. In the case of a circular crosssection, it confirms the present result that the axial strain is uniform in the core and in the shell, and that the inplane strain is also uniform in the core. Using the appropriate values of the area ratio, and the stiffness constant values of $\mathrm{GaP}[30]$ with an average ratio $\chi=1.14$ for GaAs-GaP, we calculate the solid lines shown in Figure 5 , in good agreement with numerical calculations. Note the small but visible bowing which is due to the different values of the stiffness constants in GaAs and GaP.

\subsubsection{InAs-based nanowires}

Similar results are obtained in InAs-InP NWs. They favorably compare (Fig. 6) with the results of numerical calculations [26]. We will come back to this system in the section on warping (4.3.2).

In GaAs-InAs NWs, the approximation of a constant Poisson ratio is not reasonable and a direct inversion of the full matrix (Eq. (10) where the $c_{i j}$ have been replaced by the $\tilde{c}_{i j}$, Eq. (29)), or the equivalent transfer matrix method, should be used.

\subsubsection{ZnTe nanowires}

Photoluminescence and cathodoluminescence have been measured on ZnTe-(Zn,Mg)Te core-shell NWs [1], with a peak at $2.31 \mathrm{eV}$, i.e., a $60 \mathrm{meV}$ redshift with respect to the exciton in bulk ZnTe; this is a large shift, larger than usually observed in strained 2D layers. In bare ZnTe NWs [32], a small $(3 \mathrm{meV})$ blueshift is observed.

The values of the deformation potentials in ZnTe are $[33,34] a=5.3 \mathrm{eV}$ and $d / \sqrt{3}=2.5 \mathrm{eV}$, and the values of the stiffness constants [35], $c_{11}=73.7 \mathrm{GPa}$, $c_{12}=42.3 \mathrm{GPa}$, and $c_{44}=32.1 \mathrm{GPa}$. Then the excitonic emission of a $\langle 111\rangle$ oriented cubic ZnTe NW is (in meV, with $f$ in \%) $E_{N W}=2381-88(1-\eta) f$ for the heavy hole and $E_{N W}=2381-44(1-\eta) f$ for the light hole. Note the large shift of the heavy-hole exciton, in sharp contrast with the case of a 2D epitaxial where the effect of the hydrostatic strain and the shear strain almost compensate. The heavy hole is the ground state, as found experimentally in reference [1]. For the NWs studied in reference [1], with $r_{c}=35 \mathrm{~nm}, r_{s}=65 \mathrm{~nm}$ and $f=1.04 \%$ corresponding to the lattice mismatch between a ZnTe core and a $\mathrm{Zn}_{0.8} \mathrm{Mg}_{0.2} \mathrm{Te}$ shell [36], we obtain $2.31 \mathrm{eV}$ for the heavyhole exciton, in agreement with the observed PL line, see Figure 7. The small blueshift observed in bare ZnTe NW was attributed to a small residual strain due to a thin oxide shell [32].

\subsection{Deviations from cylindrical symmetry}

In this section we discuss the two simplifying assumptions which allow us to derive the previous analytical expressions: (1) NWs have a circular cross-section, and (2) in the NWs with the zinc-blende structure, the deviation from cylindrical symmetry is small.

\subsubsection{Facets}

Most of the numerical calculations consider NWs with an hexagonal cross-sections, and actual NWs exhibit more or less well-defined facets. The present calculation does not reproduce the inhomogeneity of the in-plane strain which is calculated for a hexagonal NW, but it was already noted [37] that the central values of strain are quite similar in hexagonal and circular cores. This was confirmed in the very detailed study of reference [6], where NWs with hexagonal and circular cross sections are compared. Indeed the results of the present model compare fairly well to the results of numerical calculations made for hexagonal NWs. Other approaches are reviewed in reference [7] for the case of nanowires embedded in an infinite or semiinfinite material.

\subsubsection{Warping terms}

The cylindrical symmetry is exact in the case of NWs with the wurtzite structure, with the $c$-axis along the NW. It is not for NWs with the zinc-blende structure. As a result, the shell is warped, as evidenced in the numerical treatment of reference [6]. We now describe the analytical calculation of this additional contribution.

Indeed, when calculating the stress corresponding to the cylindrical strain configuration, additional components appear through the $\tilde{c}_{14}$ terms in the stiffness tensor: for instance, at the interface in the $y$-direction $(x=0$, $\left.y=r_{c}\right)$, a stress component normal to the interface and surface, $\sigma_{y z}=\tilde{c}_{14}\left(\varepsilon_{x x}-\varepsilon_{y y}\right)+2 \tilde{c}_{44} \varepsilon_{z y}$, takes a finite value if we use the strain of equations (18) and (32), or (25) and (33). We thus expect an additional strain to appear, 


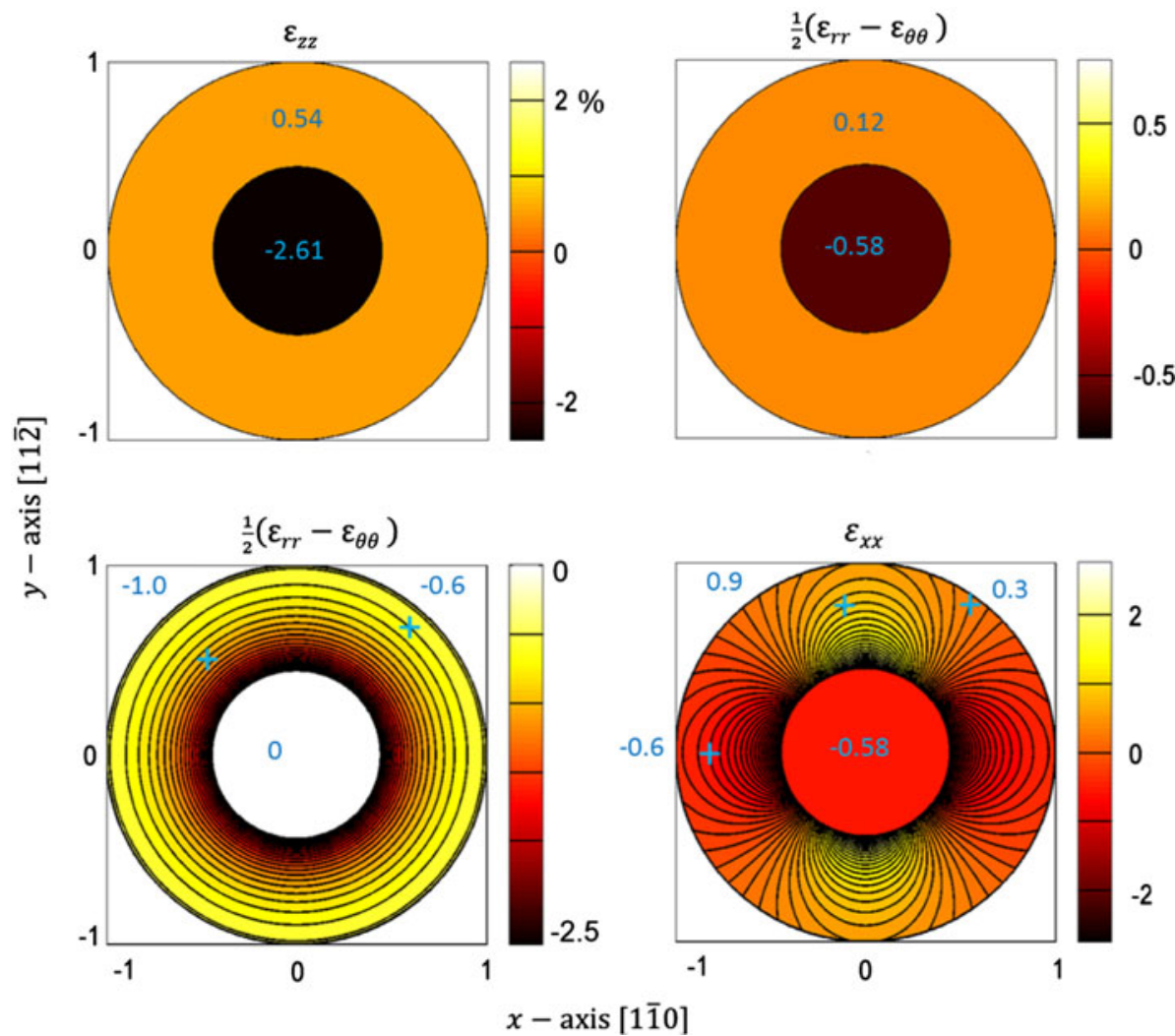

Fig. 6. Strain maps for an InAs core with an InP shell, with the same area ratio $\eta=0.2$ as in reference [26]. The lattice mismatch is $f=-3.15 \%$, stiffness ratio $\chi=1.2$, and $B_{s} \tilde{c}_{14} / \tilde{c}_{44}=-0.82 \%$. Contour line spacings are $0.1 \%$. The shear component $\varepsilon_{z z}$ in (a) and $\frac{1}{2}\left(\varepsilon_{x x}+\varepsilon_{y y}\right)=\frac{1}{2}\left(\varepsilon_{r r}+\varepsilon_{\theta \theta}\right)$ in (b) are uniform in the core and in the shell. A shear strain $\frac{1}{2}\left(\varepsilon_{r r}-\varepsilon_{\theta \theta}\right)$ is present in the shell; it is rotationally invariant (c). Plotting $\varepsilon_{x x}$ introduces an apparent dependence on $\theta$. Maps of $\varepsilon_{x x}-\varepsilon_{y y}$, $2 \varepsilon_{x y}$ and $\varepsilon_{y y}-\varepsilon_{x x}$ are identical, but for a rotation by $\pi / 4$ or $\pi / 2$. Maps (a), (b) and (d) can be compared to reference [26].

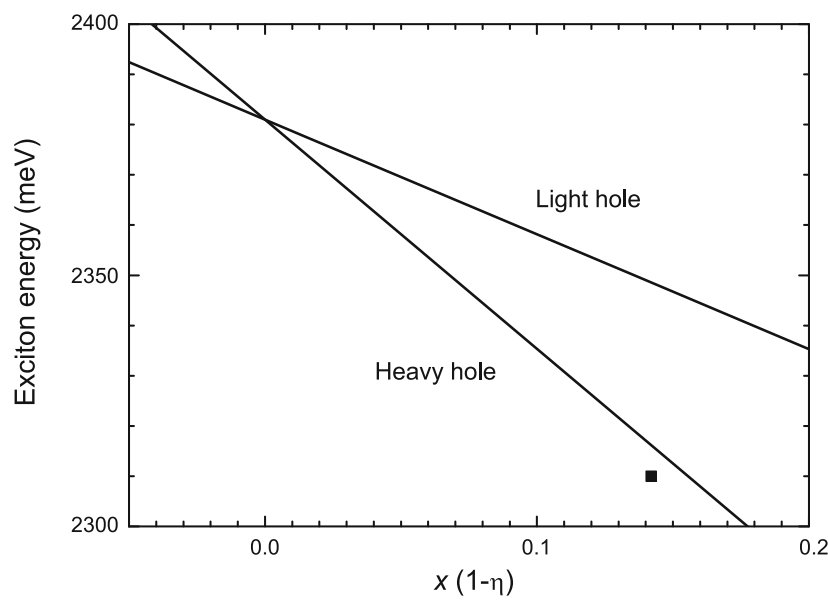

Fig. 7. Exciton energy in a $\mathrm{ZnTe} \mathrm{NW}$ with a $(\mathrm{Zn}, \mathrm{Mg}) \mathrm{Te}$ shell, as a function of area ratio $(1-\eta)$ times the $\mathrm{Mg}$ content $x$. Confinement effects are ignored.

$\varepsilon_{z y}=-\tilde{c}_{14}\left(\varepsilon_{x x}-\varepsilon_{y y}\right) / 2 \tilde{c}_{44}$ where $\left(\varepsilon_{x x}-\varepsilon_{y y}\right)$ is taken from equation (18) and (32) or (25) and (33): it vanishes in the core (where $B_{c}=0$ ), but not in the shell where a nonuniform shear strain $\left(\varepsilon_{\theta \theta}^{s}-\varepsilon_{r r}^{s}\right)$ exists. With $c<0$, and $f<0$ (case of GaAs-GaP, InAs-GaAs, CdTe-ZnTe coreshell NWs, not ZnTe-(Zn,Mg)Te), we expect a positive $\varepsilon_{z y}$, i.e., the shell is pushed upward, towards [1 111 . Note that other non-vanishing stress components are obtained by re-introducing these warping terms into the calculation of the stress, so that they are of second-order in $\tilde{c}_{14}$.

Using the rotated stiffness tensor, equation (30), and forcing the stress component $\sigma_{r z}$ to be zero (and neglecting a contribution of second-order in $\tilde{c}_{14}$ ), we obtain $\varepsilon_{z r}=$ $\left(\tilde{c}_{14} / 2 \tilde{c}_{44}\right)\left(\varepsilon_{\theta \theta}-\varepsilon_{r r}\right) \sin 3 \theta$ : the shell is alternately pushed upward and downward, with the expected trigonal symmetry (Fig. 8).

To calculate the complete strain distribution, we must re-calculate the displacement field thanks to the LaméClapeyron-Navier equation.

When introducing the cylindrical solution $u_{r}(r)=$ $\left.A r+B r_{c}^{2} / r, u_{\theta}=0, u_{z}(z)\right)$ into the complete equation, equation (31), the $\tilde{c}_{14}$ terms vanish everywhere but in the third equation for the shell. There, $2 \partial^{2} u_{x} / \partial x \partial y+$ $\partial^{2} u_{y} / \partial x^{2}-\partial^{2} u_{y} / \partial y^{2}=8 B_{s} r_{c}^{2} \sin 3 \theta / r^{3}$. In a treatment to first-order in $\tilde{c}_{14}$ (i.e, in the cubic anisotropy $c$ ), we have to find an additional displacement $\delta \mathbf{u}$ which is solution of the Lamé-Clapeyron-Navier equation for the transversely isotropic NW, with no lattice mismatch (they are already compensated) but with body forces $F_{x}=0, F_{y}=0$, $F_{z}=\tilde{c}_{14} 8 B_{s} r_{c}^{2} \sin 3 \theta / r^{3}$ in the shell.

Thus, $\delta \mathbf{u}$ is the response of an isotropic system to an axial shear strain [9] of trigonal symmetry $(\sim \sin 3 \theta)$. 


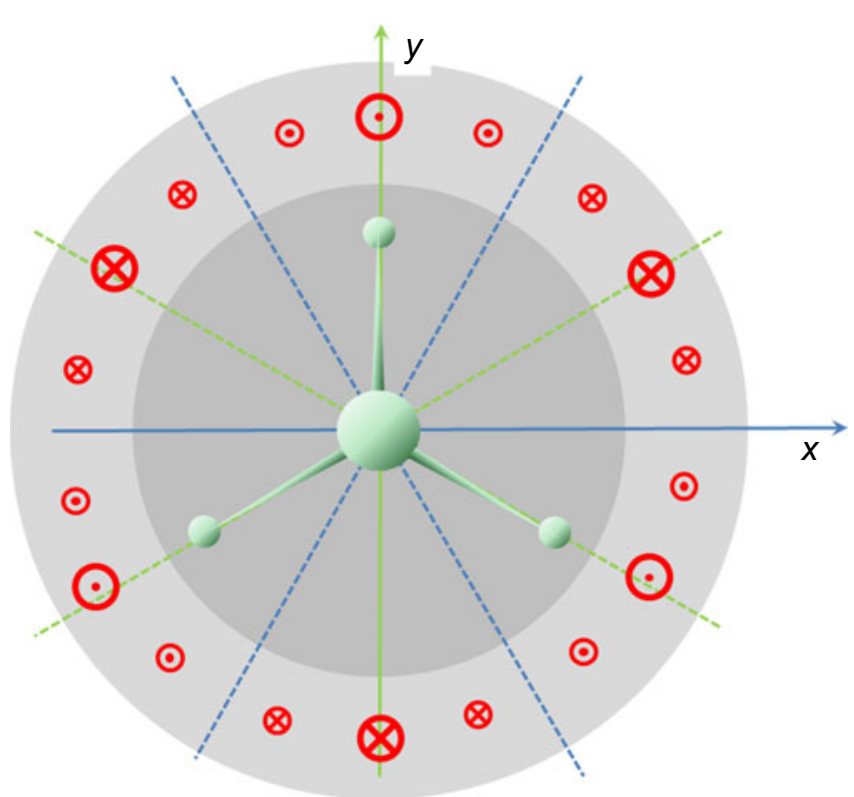

Fig. 8. Warping body forces in the shell of a core-shell NW with $f<0$, grown along $\langle 111\rangle$, due to the trigonal symmetry of the zinc-blende structure. The shell is pushed upward and downward according to the red arrows. The tetrahedron of the atomic structure is shown in green.

The solution is $\delta u_{x}=0, \delta u_{y}=0$ and $\delta u_{z}$ such that:

$$
\begin{aligned}
& \tilde{c}_{44}\left(\frac{\partial^{2}}{\partial x^{2}}+\frac{\partial^{2}}{\partial y^{2}}\right) \delta u_{z}=0 \\
& \tilde{c}_{44}\left(\frac{\partial^{2}}{\partial x^{2}}+\frac{\partial^{2}}{\partial y^{2}}\right) \delta u_{z}+\tilde{c}_{14} 8 B_{s} r_{c}^{2} \frac{\sin 3 \theta}{r^{3}}=0
\end{aligned}
$$

in the core and in the shell, respectively. The result is of trigonal symmetry and can be written, respectively (see Appendix B for details):

$$
\begin{aligned}
\frac{\delta u_{z}}{r_{c}} & =\frac{\tilde{c}_{14}}{\tilde{c}_{44}} B_{s} \alpha_{3}^{c} \frac{r^{3}}{r_{c}^{3}} \sin 3 \theta \\
\frac{\delta u_{z}}{r_{c}} & =\frac{\tilde{c}_{14}}{\tilde{c}_{44}} B_{s}\left(\frac{r_{c}}{r}+\alpha_{3}^{s} \frac{r^{3}}{r_{c}^{3}}+\alpha_{-3}^{s} \frac{r_{c}^{3}}{r^{3}}\right) \sin 3 \theta,
\end{aligned}
$$

where we have used $\alpha_{-3}^{c}=0$ (no diverging term), and the three parameters $\alpha_{3}^{c}, \alpha_{3}^{s}, \alpha_{-3}^{s}$ are determined by the boundary conditions: the non-trivial boundary conditions are that $\delta u_{z}$ and $\sigma_{r z}=\tilde{c}_{14} \sin 3 \theta\left(\varepsilon_{r r}-\varepsilon_{\theta \theta}\right)+$ $\tilde{c}_{44} \frac{1}{2} \partial\left(\delta u_{z}\right) / \partial r$ are continuous at the interface, and $\sigma_{r z}$ vanishes at the surface. The final result is:

$$
\begin{aligned}
{\left[\frac{\delta u_{z}}{r_{c}}\right]_{c} } & =\left[\eta^{2}(1-\eta) \frac{r^{3}}{r_{c}^{3}}\right] \frac{\tilde{c}_{14}}{\tilde{c}_{44}} B_{s} \sin 3 \theta \\
{\left[\varepsilon_{r z}\right]_{c} } & =\frac{3}{2}\left[\eta^{2}(1-\eta) \frac{r^{2}}{r_{c}^{2}}\right] \frac{\tilde{c}_{14}}{\tilde{c}_{44}} B_{s} \sin 3 \theta \\
{\left[\varepsilon_{\theta z}\right]_{c} } & =\frac{3}{2}\left[\eta^{2}(1-\eta) \frac{r^{2}}{r_{c}^{2}}\right] \frac{\tilde{c}_{14}}{\tilde{c}_{44}} B_{s} \cos 3 \theta \\
{\left[\frac{\delta u_{z}}{r_{c}}\right]_{s} } & =\left[\frac{r_{c}}{r}-\frac{r_{c}^{3}}{r^{3}}+\eta^{2}(1-\eta) \frac{r^{3}}{r_{c}^{3}}\right] \frac{\tilde{c}_{14}}{\tilde{c}_{44}} B_{s} \sin 3 \theta
\end{aligned}
$$

$$
\begin{aligned}
& {\left[\varepsilon_{r z}\right]_{s}=\frac{3}{2}\left[-\frac{r_{c}^{2}}{3 r^{2}}+\frac{r_{c}^{4}}{r^{4}}+\eta^{2}(1-\eta) \frac{r^{2}}{r_{c}^{2}}\right] \frac{\tilde{c}_{14}}{\tilde{c}_{44}} B_{s} \sin 3 \theta} \\
& {\left[\varepsilon_{\theta z}\right]_{s}=\frac{3}{2}\left[\frac{r_{c}^{2}}{r^{2}}-\frac{r_{c}^{4}}{r^{4}}+\eta^{2}(1-\eta) \frac{r^{2}}{r_{c}^{2}}\right] \frac{\tilde{c}_{14}}{\tilde{c}_{44}} B_{s} \cos 3 \theta,}
\end{aligned}
$$

where

$$
\begin{aligned}
& \frac{\tilde{c}_{14}}{\tilde{c}_{44}} B_{s}=-\frac{f}{\sqrt{2}} \frac{c_{11}-c_{12}-2 c_{44}}{c_{11}-c_{12}+c_{44}} \times \\
& \frac{3\left(c_{11}+2 c_{12}\right)}{\left(c_{11}-c_{12}+4 c_{44}\right)[\eta+(1-\eta) \chi]+\left(2 c_{11}+4 c_{12}+2 c_{44}\right)} .
\end{aligned}
$$

The results are shown in Figure 9 for an InAs-InP NW with the area ratio of reference [26], and for a thick shell. Maps are shown in Figure 10. Note the discontinuity of $\varepsilon_{r z}$ at the interface, and its fast decay while $\varepsilon_{\theta z}$ progressively increases from zero and stays finite far into the shell. There is a complete agreement with the results of numerical calculations in reference [26].

Apart from the presence of this additional shear strain, the other strain components are modified by terms of the order of $\left(\tilde{c}_{14} / \tilde{c}_{11}\right)^{2}$. Taking again GaAs parameters, we find that these second order terms are of the order of $1 \% \times f$. As a result, the change of the core strain induced by the $\tilde{c}_{14}$ terms is negligible. Note also that the contribution of the additional shear strain to $\sigma_{z z}$ vanishes due to the $\cos 3 \theta$ and $\sin 3 \theta$ factors.

\subsection{Summary and electronic properties}

To sum up, the strain configuration in a zinc-blende NW grown along the $\langle 111\rangle$ axis is described by a cylindrical strain, equation (18) and (32) (or Eqs. (25) and (33) if $\chi=$ 1 ), complemented by an axial shear strain ("warping"), equation (37).

The Bir-Pikus Hamiltonian describing the coupling of holes to strain has the same symmetry as the Luttinger Hamiltonian. When expressed in the present trigonal basis (hole states $\left|\frac{3}{2}\right\rangle,\left|\frac{1}{2}\right\rangle,\left|-\frac{1}{2}\right\rangle$ and $\left|-\frac{3}{2}\right\rangle$ quantized along [1 111$]$,

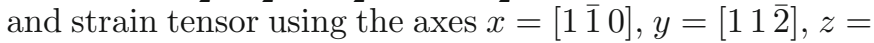
[1 111$]$ ), using the symmetry arguments of reference [38] as described in reference [39], the Hamiltonian writes:

$$
\left(\begin{array}{cccc}
P+Q & -S & R & 0 \\
-S^{*} & P-Q & 0 & R \\
R^{*} & 0 & P-Q & S \\
0 & R^{*} & S^{*} & P+Q
\end{array}\right)
$$

with

$$
\begin{aligned}
P= & -a\left(\varepsilon_{x x}+\varepsilon_{y y}+\varepsilon_{z z}\right) \\
Q= & \frac{d}{2 \sqrt{3}}\left(\varepsilon_{x x}+\varepsilon_{y y}-2 \varepsilon_{z z}\right) \\
R= & -\frac{\sqrt{3}}{6}\left(b+\frac{2 d}{\sqrt{3}}\right)\left(\varepsilon_{x x}-\varepsilon_{y y}-2 i \varepsilon_{x y}\right) \\
& +\frac{2}{\sqrt{6}}\left(b-\frac{d}{\sqrt{3}}\right)\left(\varepsilon_{x z}+i \varepsilon_{y z}\right)
\end{aligned}
$$


The European Physical Journal Applied Physics
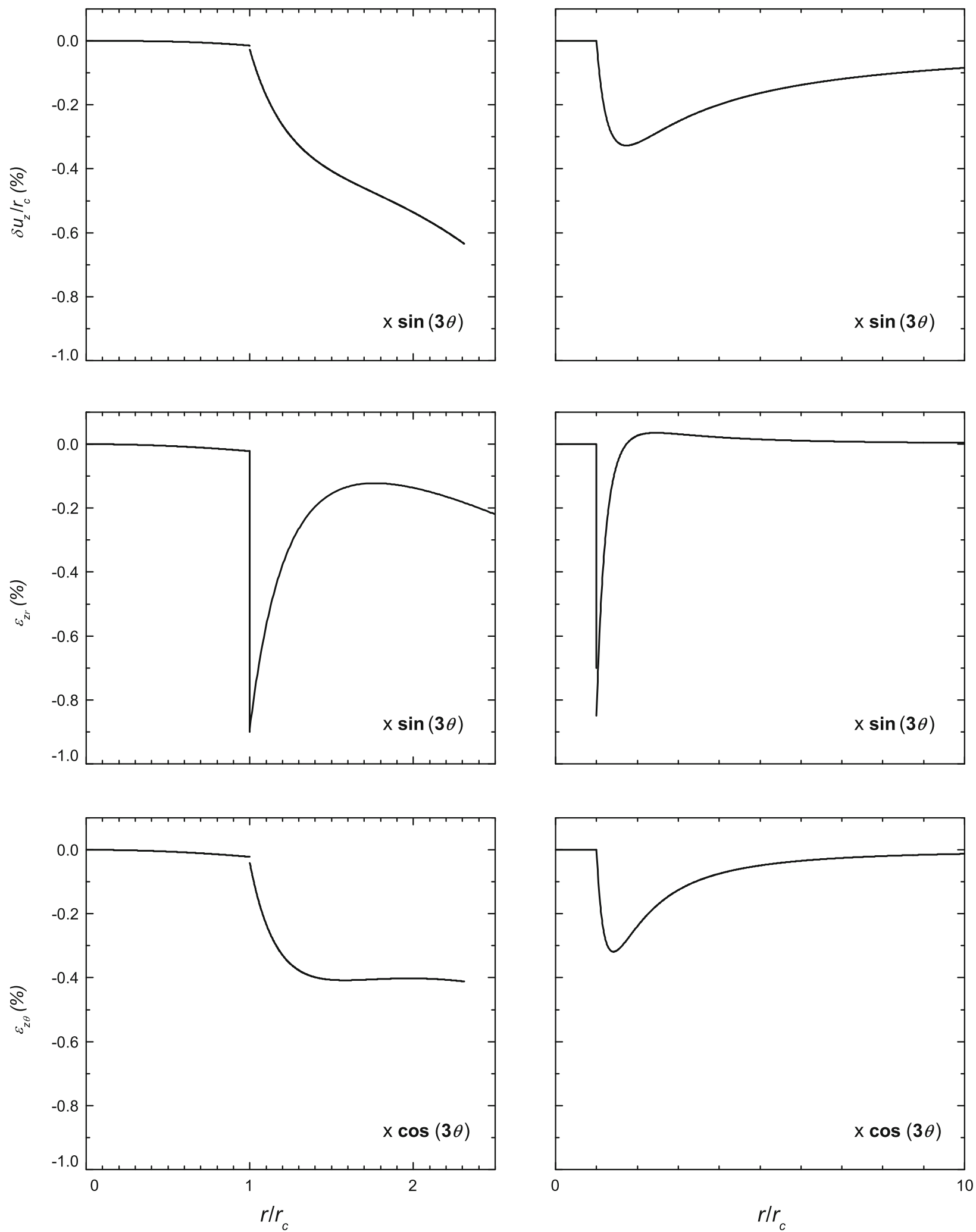

Fig. 9. Warping strain due to the trigonal symmetry of the zinc-blende structure in an InAs-InP core shell NW. The lattice mismatch is $f=-3.15 \%$, stiffness ratio $\chi=1.2$, and $B_{s} \tilde{c}_{14} / \tilde{c}_{44}=-0.82 \%$. The radial dependence of the displacement along the axis (top), the radial-axial shear strain (middle) and the tangential-axial shear strain (bottom) are shown for a NW with finite shell radius (left column)and infinite shell radius (right column). The trigonal symmetry appears through the sin $3 \theta$ or $\cos 3 \theta$ factor, as indicated. 


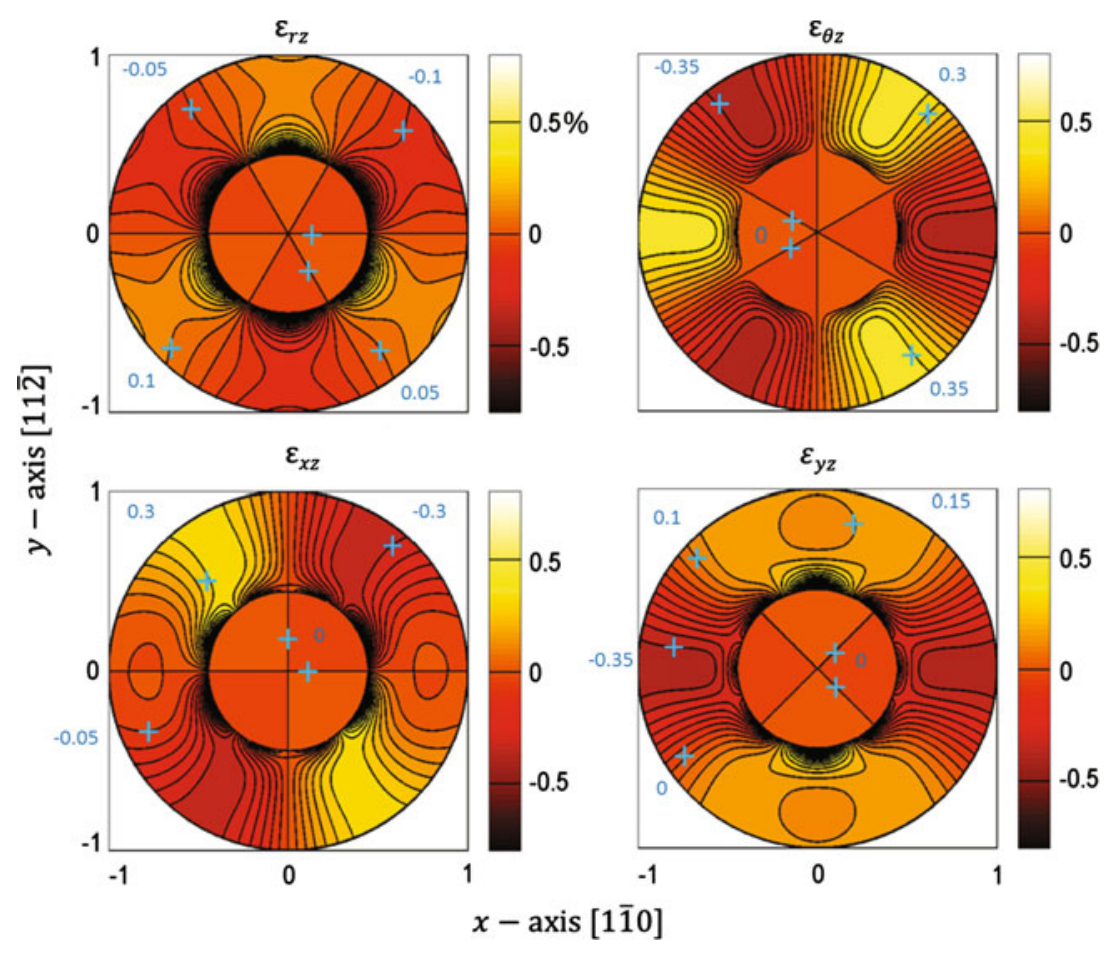

Fig. 10. Strain map for an InAs core with an $\mathrm{InP}$ shell, with the same area ratio $\eta=0.2$ as in reference [26]. The lattice mismatch is $f=-3.15 \%$, stiffness ratio $\chi=1.2$, and $B_{s} \tilde{c}_{14} / \tilde{c}_{44}=-0.82 \%$. All contour line spacings are $0.05 \%$. The shear components $\varepsilon_{r z}$ and $\varepsilon_{\theta z}$ reveal the trigonal symmetry. This symmetry is masked if we plot $\varepsilon_{x z}$ or $\varepsilon_{x z}$ but these plots can be compared qualitatively to reference [6], and quantitatively (but taking into account the different axes used) to reference [26].

$$
\begin{aligned}
S= & \frac{\sqrt{3}}{3}\left(2 b+\frac{d}{\sqrt{3}}\right)\left(\varepsilon_{x z}-i \varepsilon_{y z}\right) \\
& -\frac{1}{\sqrt{6}}\left(b-\frac{d}{\sqrt{3}}\right)\left(\varepsilon_{x x}-\varepsilon_{y y}+2 i \varepsilon_{x y}\right) .
\end{aligned}
$$

In the core, apart from a small axial shear strain, which takes non-vanishing values close to the interface but remains very small, the strain comprises the hydrostatic strain $\left(\varepsilon_{x x}+\varepsilon_{y y}+\varepsilon_{z z}\right)$ and the trigonal strain $\left(\varepsilon_{x x}+\right.$ $\left.\varepsilon_{y y}-2 \varepsilon_{z z}\right)$. The Bir-Pikus Hamiltonian is diagonal in the trigonal basis, with a splitting equal to $2 Q$; the Luttinger Hamiltonian gives the effective masses of the eigenstates: the mass along the NW axis (determining the density of states and transport properties) is $m^{*}=m_{0} /\left(\gamma_{1}-2 \gamma_{3}\right)$ for the $\left| \pm \frac{3}{2}\right\rangle$ holes, and $m^{*}=m_{0} /\left(\gamma_{1}+2 \gamma_{3}\right)$ for the $\left| \pm \frac{1}{2}\right\rangle$ holes, the mass in the plane (governing confinement) being $m^{*}=m_{0} /\left(\gamma_{1} \pm \gamma_{3}\right)$. This was used in Section 4.1.2.

In the shell, close to the interface, the dominant contribution is the shear strain with cylindrical symmetry: it adds non-diagonal matrix elements $(R$ and $S)$ to the BirPikus Hamiltonian, which mixes the previous states. As in the previous case of a wurtzite NW, half of the holes are confined to the interface. If we consider the whole NW, the axial symmetry is preserved, so that the eigenstates in the core retain their symmetry, with some mixing expected to take place in narrow NWs. However, there is also a contribution from the warping terms in the shell, which adds a modulation with a three-fold symmetry to the hole potential: this complex structure may contribute to localization, particularly in NWs with a thick shell.

This deformation potential landscape is complemented by the piezoelectric effect [26]. Again, the polarization in the core is along the axis, determined by $-e_{14}\left(\varepsilon_{x x}+\right.$ $\left.\varepsilon_{y y}-2 \varepsilon_{z z}\right) / \sqrt{3}$ where $e_{14}$ is the unique coefficient of the piezoelectric tensor (the indices refer to the cubic axes). A complex lanscape however emerges in the shell from the presence of in-plane and axial shear strains, and of additional terms in the piezoelectric tensor written in the trigonal axes $[3,26,27]$.

\section{Cubic semiconductors along $\langle 001\rangle$}

By contrast to the (111) plane of the zinc-blende structure, which is quite isotropic, the (001) plane is known to be strongly anisotropic. This is obvious on the stiffness tensor written in the $\mathbf{e}_{r}, \mathbf{e}_{\theta}, \mathbf{e}_{z}$ axes, obtained by rotating the cubic axes by an angle $\theta$ ) around $z$ (i.e., it is written in cylindrical coordinates):

$$
\left(\begin{array}{cccccc}
\hat{c}_{11}+\frac{c \cos 4 \theta}{4} & \hat{c}_{12}-\frac{c \cos 4 \theta}{4} & \hat{c}_{13} & 0 & 0 & 0 \\
\hat{c}_{12}-\frac{c \cos 4 \theta}{4} & \hat{c}_{11}+\frac{c \cos 4 \theta}{4} & \hat{c}_{13} & 0 & 0 & 0 \\
\hat{c}_{13} & \hat{c}_{13} & \hat{c}_{33} & 0 & 0 & 0 \\
0 & 0 & 0 & \hat{c}_{44} & 0 & 0 \\
0 & 0 & 0 & 0 & \hat{c}_{44} & 0 \\
0 & 0 & 0 & 0 & 0 & \hat{c}_{66}-\frac{c \cos 4 \theta}{4}
\end{array}\right)
$$


with

$$
\begin{aligned}
& \hat{c}_{11}=\frac{3 c_{11}+c_{12}+2 c_{44}}{4} \\
& \hat{c}_{12}=\frac{c_{11}+3 c_{12}-2 c_{44}}{4} \\
& \hat{c}_{66}=\frac{c_{11}-c_{12}+2 c_{44}}{4} \\
& \hat{c}_{13}=c_{12}, \hat{c}_{33}=c_{11}, \hat{c}_{44}=c_{44} .
\end{aligned}
$$

Reference [40] proposes a solution where one assumes the cylindrical form of the displacement field, $u_{r}(r)=A r+$ $B / r$, and writes boundary conditions at the interfaces/ surfaces only in the cubic directions. Actually, as we show now, the cylindrical displacement field is not a solution of the Lamé-Clapeyron-Navier equation, and the boundary conditions are not valid for other directions of the basal plane. We thus propose a solution in two steps, along the line we followed in the previous section for the NW with trigonal axis. We thus identify the stiffness constants which give the better approximation by a cylindrical solution, and we calculate the additional strain with fourfold symmetry, which is now a generalized in-plane shear strain.

The present form of the stiffness tensor, equation (39), identifies two contributions:

- one with cylindrical symmetry (that with the $\hat{c}$, note that $\hat{c}_{11}-\hat{c}_{12}=2 \hat{c}_{66}$ ); if we keep only this contribution, the strain configuration is that of equation (18) or equation $(25)$, where the $c_{i j}$ 's are replaced by the $\hat{c}_{i j}$ 's;

- one, proportional to $c$, with the expected fourfold symmetry around $z$. As mentioned earlier, with $c<0$, a zinc-blende crystal is softer against a pure tetragonal stress (along a cubic axis, $\cos 4 \theta=1$ ) than against any other stress, in particular along a $\langle 110\rangle$ axis $(\cos 4 \theta=-1)$.

Omitting the terms violating the translation invariance and identifying cylindrical contributions (in $\hat{c}$ ) and contributions due to the cubic anisotropy (proportional to $c$ ), we obtain for the Lamé-Clapeyron-Navier equation in Cartesian coordinates:

$$
\begin{gathered}
\frac{\hat{c}_{11}-\hat{c}_{12}}{2}\left(\frac{\partial^{2}}{\partial x^{2}}+\frac{\partial^{2}}{\partial y^{2}}\right) u_{x} \\
+\frac{\hat{c}_{11}+\hat{c}_{12}}{2} \frac{\partial}{\partial x}\left(\frac{\partial u_{x}}{\partial x}+\frac{\partial u_{y}}{\partial y}\right) \\
+\frac{c}{4}\left(\frac{\partial^{2} u_{x}}{\partial x^{2}}-\frac{\partial^{2} u_{x}}{\partial y^{2}}-2 \frac{\partial^{2} u_{y}}{\partial x \partial y}\right)=0 \\
\frac{\hat{c}_{11}-\hat{c}_{12}}{2}\left(\frac{\partial^{2}}{\partial x^{2}}+\frac{\partial^{2}}{\partial y^{2}}\right) u_{y} \\
+\frac{\hat{c}_{11}+\hat{c}_{12}}{2} \frac{\partial}{\partial y}\left(\frac{\partial u_{x}}{\partial x}+\frac{\partial u_{y}}{\partial y}\right) \\
+\frac{c}{4}\left(\frac{\partial^{2} u_{y}}{\partial y^{2}}-\frac{\partial^{2} u_{y}}{\partial x^{2}}-2 \frac{\partial^{2} u_{x}}{\partial x \partial y}\right)=0
\end{gathered}
$$

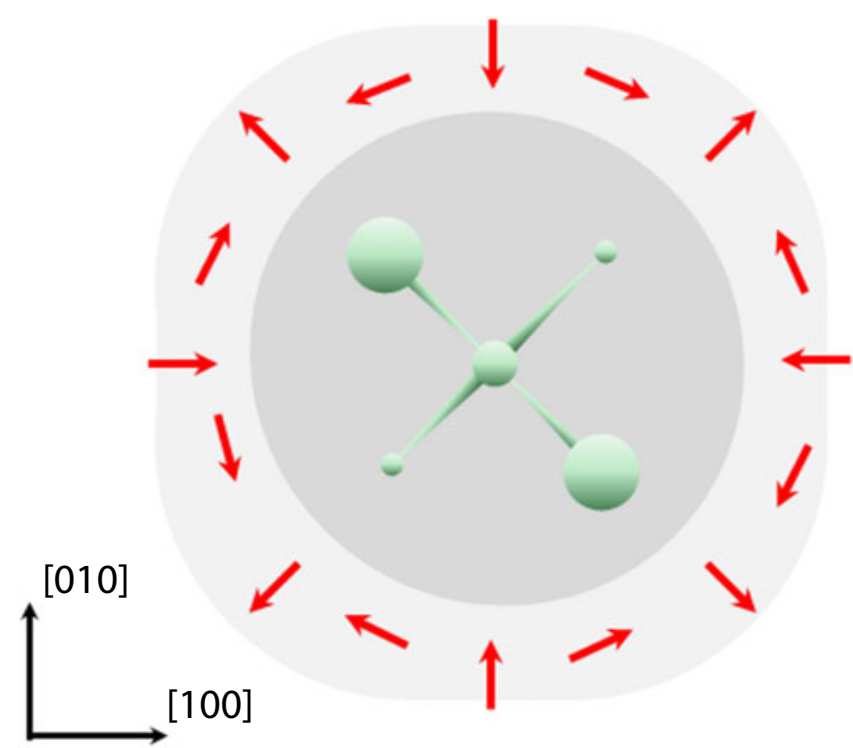

Fig. 11. In-plane body forces in the shell of a $\langle 001\rangle$ NW, with $f<0$, due to the four-fold symmetry of the zinc-blende structure. The tetrahedron of the atomic structure is shown in green.

$$
\frac{\partial^{2} u_{z}}{\partial x^{2}}+\frac{\partial^{2} u_{z}}{\partial y^{2}}=0 .
$$

Inserting the cylindrical solution $u_{r}(r)=A r+B / r$ reveals non-vanishing contributions from the terms proportional to $c$. As in the previous case, in a calculation to first-order in $c$, these terms act as body forces and generate an additional displacement field $\delta \mathbf{u}$, proportional to $c$. Even if these terms look quite similar to those already encountered for the $\langle 111\rangle \mathrm{NW}$ (they amount to $8 B r_{c}^{2} \sin 3 \theta / r^{3}$ for the first equation of Eq. (40) and $-8 B r_{c}^{2} \cos 3 \theta / r^{3}$ for the second one), they appear as body forces in the basal plane, organized as a transverse shear stress [9] with fourfold symmetry:

$$
\begin{aligned}
& F_{r}=c \frac{2 B_{s} r_{c}^{2}}{r^{3}} \cos 4 \theta \\
& F_{\theta}=-c \frac{2 B_{s} r_{c}^{2}}{r^{3}} \sin 4 \theta
\end{aligned}
$$

in the shell, and zero in the core (Fig. 11). We thus have to find an additional in-plane displacement $\delta \mathbf{u}(r, \theta)$ which is the response of the transversely isotropic system to these forces. The relevant part of the Lamé-Clapeyron-Navier equation is a two-dimensional equation:

$$
\frac{\hat{c}_{11}-\hat{c}_{12}}{2} \triangle \delta \mathbf{u}+\frac{\hat{c}_{11}+\hat{c}_{12}}{2} \nabla(\nabla \cdot \delta \mathbf{u})=\mathbf{F},
$$

or, defining a Poisson ratio $\nu=\hat{c}_{12} /\left(\hat{c}_{11}+\hat{c}_{12}\right)=\left(c_{11}+\right.$ $\left.3 c_{12}-2 c_{44}\right) / 4\left(c_{11}+c_{12}\right)$,

$$
(1-2 \nu) \triangle \delta \mathbf{u}+\nabla(\nabla \cdot \delta \mathbf{u})=\frac{2 \mathbf{F}}{\hat{c}_{11}+\hat{c}_{12}} .
$$




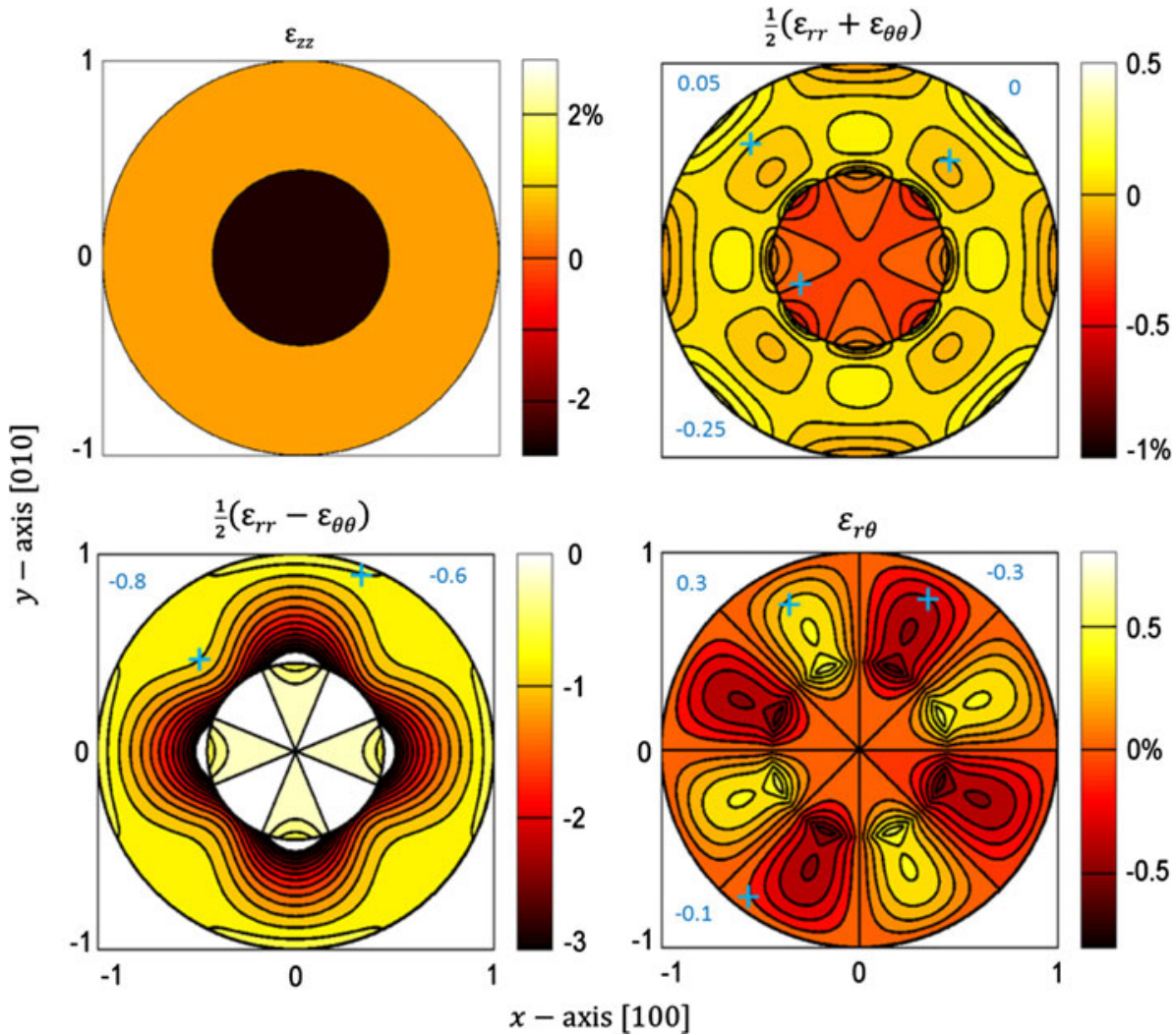

Fig. 12. Strain maps for an InAs core with an $\mathrm{InP}$ shell, with the same area ratio $\eta=0.2$ as in reference [26]. The NW axis is $\langle 001\rangle$ and we used $\chi=1$. The mismatch is $f=-3.15 \%$ and $B_{s} c /\left(c_{11}+c_{12}\right)=-0.87 \%$. The axial strain component $\varepsilon_{z z}$ is uniform. The contour line spacing is $0.05 \%$ for $\frac{1}{2}\left(\varepsilon_{r r}+\varepsilon_{\theta \theta}\right), 0.2 \%$ for $\frac{1}{2}\left(\varepsilon_{r r}-\varepsilon_{\theta \theta}\right)$, and $0.1 \%$ for $\varepsilon_{r \theta}$. All in-plane strain components exhibit a four-fold contribution due to the crystal anisotropy.

The solution is:

$$
\begin{aligned}
\frac{\delta u_{r}}{r_{c}} & =\frac{c}{\hat{c}_{11}+\hat{c}_{12}} B_{s} g_{r}(r) \cos 4 \theta \\
\frac{\delta u_{\theta}}{r_{c}} & =\frac{c}{\hat{c}_{11}+\hat{c}_{12}} B_{s} g_{\theta}(r) \sin 4 \theta,
\end{aligned}
$$

where $g_{r}$ and $g_{\theta}$ are two dimensionless functions of $r / r_{c}-$ more precisely they are sums of five terms in $\left(r / r_{c}\right)^{n}$ with $n=-1, \pm 3, \pm 5$, which are given in Appendix B.

To sum up, the strain configuration in a core-shell NW grown along $\langle 001\rangle$ is given by equation (18), with:

$$
\begin{aligned}
B_{s} & =-\frac{c_{11}+2 c_{12}}{\left(\frac{c_{11}-c_{12}}{2}+c_{44}\right)[\eta+(1-\eta) \chi]+\left(c_{11}+c_{12}\right)} f \\
f_{\perp}+B_{s} & =\frac{\left(\frac{c_{11}-c_{12}}{2}+c_{44}\right)[\eta+(1-\eta) \chi]-c_{12}}{\left(\frac{c_{11}-c_{12}}{2}+c_{44}\right)[\eta+(1-\eta) \chi]+\left(c_{11}+c_{12}\right)} f .
\end{aligned}
$$

If the materials have the same hardness $(\chi=1)$, this reduces to equation (25) and,

$$
\begin{aligned}
B_{s} & =-\frac{2\left(c_{11}+2 c_{12}\right)}{3 c_{11}+c_{12}+2 c_{44}} f \\
f_{\perp}+B_{s} & =\frac{c_{11}-3 c_{12}+2 c_{44}}{3 c_{11}+c_{12}+2 c_{44}} f .
\end{aligned}
$$

This is complemented by an in-plane shear strain which writes (for $\chi=1$ ):

$$
\begin{aligned}
& \varepsilon_{r r}=\frac{c}{c_{11}+c_{12}} B_{s} \cos 4 \theta g_{r r}\left(\frac{r}{r_{c}}\right) \\
& \varepsilon_{r \theta}=\frac{c}{c_{11}+c_{12}} B_{s} \cos 4 \theta g_{r \theta}\left(\frac{r}{r_{c}}\right) \\
& \varepsilon_{\theta \theta}=\frac{c}{c_{11}+c_{12}} B_{s} \sin 4 \theta g_{\theta \theta}\left(\frac{r}{r_{c}}\right),
\end{aligned}
$$

where $g_{\theta \theta}=4 g_{\theta}+g_{r}$, and $g_{r}, g_{\theta}, g_{r \theta}$ and $g_{r r}$ are given in Appendix B, equation (B.6) with the coefficients given in (B.9) for the shell and the core.

Figure 12 shows the strain map for an InAs-InP NW with the same area ratio $\eta$ as in Figures 6 and 10, and reference [26], but with the NW axis along $\langle 001\rangle$. Figure 13 displays the radial profiles of the in-plane displacement field, the in-plane strain components (the cylindrical contribution and the modulation in $\sin 4 \theta$ or $\cos 4 \theta$ due to cubic anisotropy), and the axial strain. The cubic contribution is negligible in the central part of the core, and remains small close to the interface; in the shell, it takes significant values, yet smaller than the cylindrical contribution. Further contributions should bring terms of higher order in $4 \theta$, with the order of magnitude of the secondorder terms around $c / 4 c_{11}$, i.e., again, a few $\%$ in GaAs. 
The European Physical Journal Applied Physics
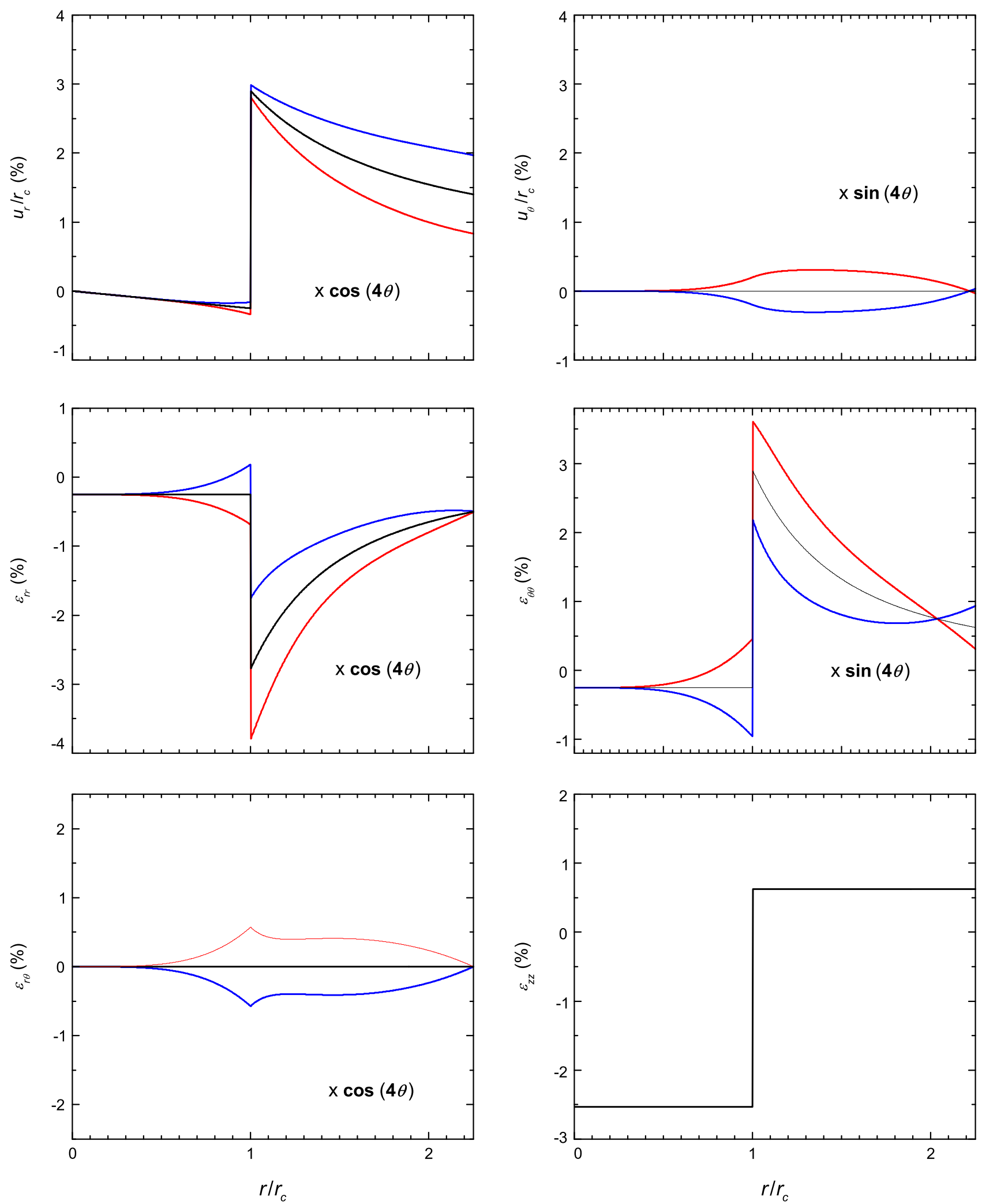

Fig. 13. Radial profile of the displacement field and the strain components for the same NW as in Figure 12 . The NW axis is $\langle 001\rangle$. The (black) central lines are the cylindrical contribution, the two other lines show the extreme values due to the cubic anisotropy, with the dependence on polar angle as indicated (blue: $\sin 4 \theta$ or $\cos 4 \theta=1$; red: $\sin 4 \theta$ or $\cos 4 \theta=-1$ ). 


\section{Discussion and conclusion}

The present study proposes an analytical treatment of the strain distribution in core-shell and multishell NWs with circular section. Several comparisons have been given with numerical treatments using either a valence force field model $[2,3,6,17,26,28]$, or a finite element implementation of continuum elasticity [26]. Even if commercial packages now exist which will give the same results as these numerical treatments, the analytical treatment remains faster, and it favors a more comprehensive understanding.

It has been recognized for a long time [13] that the (111) plane of a cubic crystal (in the present case, zincblende or diamond semiconductor) is isotropic - and the same property also holds for the $(a, b)$ plane of the wurtzite structure.

In a core-shell NW, this remains valid for a core-shell NW oriented along the $c$-axis of the wurtzite structure. This transverse isotropy has several consequences which are reminiscent from the case of a fully isotropic material.

- the longitudinal strain is decoupled from the in-plane strain. It is uniform in the core and in the shell, and results from a sharing of the lattice mismatch along the $c$ axis, inversely proportional to the cross-section areas;

- the in-plane strain in the core is isotropic and uniform. It is the result of a partial compensation between the direct effect of the in-plane lattice mismatch (the $c_{13} f_{\perp}$ contribution in Eq. (18) and the Poisson effect from the longitudinal mismatch (the $\left(c_{11}-c_{12}\right) f_{\|}$contribution). The simple result obtained for a fully isotropic material (a factor of $-\frac{1}{2}$ in the (shear strain)/(isotropic strain) ratio when comparing the NW to the thin layer) must be adapted to the relevant stiffness constants. In the case of a GaN-AlN NW, the compensation is reinforced by the different values of the lattice mismatch in the two directions, so that the in-plane strain in the core is reduced by one order of magnitude;

- actually the main part of the in-plane lattice mismatch is accommodated by the in-plane shear strain, which rotates around the interface so that the circular symmetry is maintained. The fact that this strain is restricted to the vicinity of the interface is a consequence of the Saint-Venant principle.

It is interesting to note that this shear strain induces a potential which can be used to confine holes in the shell in the vicinity of the interface, far from the sidewall. It thus allows the design of type-II core-shell NWs where both the electrons (in the core) and the holes (in the shell) are kept away from surface defects.

The strain distribution in a NW oriented along the $\langle 111\rangle$ axis of a semiconductor with the zinc-blende (or diamond) structure is more complex. Shear strains and shear stresses are expected, and they appear in the numerical studies. They are due to the trigonal symmetry around the $\langle 111\rangle$ axis, and more precisely to the presence of tetrahedral building blocks with a single orientation - while two orientations co-exist in the wurtzite structure [41]. The present analysis shows that these shear strain indeed exist in the shell, and that their influence on the strain in the core is small. The uniform strain, isotropic in the plane, which exists in the core can be calculated analytically using the stiffness tensor appropriate for the $\langle 111\rangle$ orientation.

The same method gives analytical results also in the case of a NW with the zinc-blende (or diamond) structure grown along a cubic axis: then in-plane strain with four-fold symmetry develops in addition to the cylindrical configuration.

Note that with these two examples (NWs grown along the trigonal or along the cubic axis of the zinc-blende/ diamond structure), we obtain the two possible types of additional generalized shear strain (axial or in-plane). NWs with other types of symmetry are expected to involve combinations of these two types of generalized shearstrain.

While the present study assumes a circular basis of the NWs, numerical studies also reveals the role of facets: for a hexagonal basis, the strain in the core is not uniform in the corners of the hexagons. An analytical method has been proposed for isotropic materials in reference [42]. Alternately, a possible extension of the present method would be to express the difference between the NW with a polygonal section and that with a circular one, as a field of body forces, which would be localized at the corners of the polygon; then, as we did for the crystalline anisotropy, we could calculate the response of the system to that field. Nevertheless, the comparison between the present calculation and the plateaus values from numerical studies suggests again a quantitative agreement, which can be seen as another consequence of the Saint-Venant's principle.

Finally, multishell NWs are currently proposed for applications such as the direct-bandgap emission from $\left\langle\begin{array}{lll}0 & 0 & 1\end{array}\right\rangle$ Si-Ge NWs [43], or a reduction of piezoelectric effects in wurtzite or $\langle 111\rangle$ zinc-blende NWs [15]. The present study shows that a shear strain exists in such lateral QWs, different from well to well. The transfer matrix method can also be used to incorporate the effects of surface stress, which may become significant in narrow NWs [44], or of surface layers (oxide for instance), two effects which will be difficult to disentangle.

This work was done in the joint CNRS-CEA group "Nanophysique \& semiconducteurs", and in the frame of the ANR project "Magwires" (ANR-11-BS10-013). We thank Yann-Michel Niquet, Moïra Hocevar and all the members of the Magwires project for many discussions and for communicating their results.

\section{References}

1. P. Wojnar, E. Janik, L.T. Baczewski, S. Kret, E. Dynowska, T. Wojciechowski, J. Suffczynski, J. Papierska, P. Kossacki, G. Karczewski, J. Kossut, T. Wojtowicz, Nano. Lett. 12, 3404 (2012)

2. Y.M. Niquet, C. Delerue, C. Krzeminski, Nano Lett. 12, 3545 (2012) 
3. F. Boxberg, N. Søndergaard, H.Q. Xu, Nano Lett. 10, 1108 (2010)

4. M. Amato, M. Palummo, S. Ossicini, Mat. Sci. Eng. B 177, $705(2012)$

5. M.Yu. Gutkin, I.A. Ovid'ko, A.G. Sheinerman, J. Phys. Condens. Matter. 12, 5391 (2000)

6. J. Grönqvist, N. Søndergaard, F. Boxberg, T. Guhr, S. Åberg, H.Q. Xu, J. Appl. Phys. 106, 53508 (2009)

7. R. Maranganti, P. Sharma, J. Comput. Theor. Nanosci. 4, 715 (2007)

8. J.F. Nye, Physical Properties of Crystals: Their Representation by Tensors and Matrices (Oxford University Press, Oxford, 1985)

9. I. Tsukrov, B. Drach, Int. J. Solids Structures 47, 25 (2010)

10. P.M. Marcus, F. Jona, Phys. Rev. B 51, 5263 (1995)

11. G.L. Bir, G. Pikus, Symmetry and Strain-Induced Effects in Semiconductors (Wiley, New York, 1974)

12. K.E. Aifantis, A.L. Kolesnikova, A.E. Romanov, J. Phil. Mag. 87, 4731 (2007)

13. J.J. Wortman, R.A. Evans, J. Appl. Phys. 36, 153 (1965)

14. C.W. Warwick, T.W. Clyne, J. Mater. Sci. 26, 3817 (1991)

15. R. Koester, J.-S. Hwang, D. Salomon, X.J. Chen, C. Bougerol, J.-P. Barnes, D. Le Si Dang, L. Rigutti, A. de Luna Bugallo, G. Jacopin, M. Tchernycheva, C. Durand, J. Eymery, Nano Lett. 11, 4839 (2011)

16. J.H. Davies, J. Appl. Phys. 84, 1358 (1998)

17. K. Herstroffer, R. Mata, D. Camacho, C. Leclere, G. Tourbot, Y.M. Niquet, A. Cros, C. Bougerol, H. Renevier, B. Daudin, Nanotechnology 21, 415702 (2010)

18. L. Rigutti, G. Jacopin, L. Largeau, E. Galopin, A. de Luna Bugallo, F.H. Julien, J.-C. Harmand, F. Glas, M. Tchernycheva, Phys. Rev. B 83, 155320 (2011)

19. A. Polian, M. Grimsditch, I. Grzegory, J. Appl. Phys. 79, 3343 (1996)

20. A.F. Wright, J. Appl. Phys. 82, 2833 (1997)

21. L.E. McNeil, M. Grimsditch, R.H. French, J. Am. Ceram. Soc. 76, 1132 (1993)

22. T. Azuhata, M. Takesada, T. Yagi, A. Shikanai, S.F. Chichibu, K. Torii, A. Nakamura, T. Sota, G. Cantwell, D.B. Eason, C.W. Litton, J. Appl. Phys. 94, 968 (2003)

23. Y.-I. Kim, K. Page, R. Seshadri, Appl. Phys. Lett. 90, 101904 (2007)

24. R. André, J. Cibert, Le Si Dang, J. Zeman, M. Zigone, Phys. Rev. B 53, 6951 (1996)

25. A. Beya-Wakata, P.-Y. Prodhomme, G. Bester, Phys. Rev. B 84, 195207 (2011)
26. F. Boxberg, N. Sondergaard, H.Q. Xu, Adv. Mater. 24 4692 (2012)

27. S. Schulz, M.A. Caro, E.P. O'Reilly, O. Marquardt, Phys. Rev. B 84, 125312 (2011)

28. M. Hocevar, L.T.T. Giang, R. Songmuang, M. den Hertog, L. Besombes, J. Bleuse, Y.-M. Niquet, N.T. Pelekanos, Appl. Phys. Lett. 102, 191103 (2013)

29. S. Adachi, J. Appl. Phys. 58, R1 (1985)

30. I. Vurgaftman, J.R. Meyer, L.R. Ram-Mohan, J. Appl. Phys. 89, 5815 (2001)

31. M. Montazeri, M. Fickenscher, L.M. Smith, H.E. Jackson, J. Yarrison-Rice, J. Hyun Kang, Q. Gao, H. Hoe Tan, C. Jagadish, Y. Guo, J. Zou, M.-E. Pistol, C.E. Pryor, Nano Lett. 10, 880 (2010)

32. A. Artioli, P. Rueda-Fonseca, P. Stepanov, E. BelletAmalric, M. Den Hertog, C. Bougerol, Y. Genuist, F. Donatini, R. André, G. Nogues, K. Kheng, S. Tatarenko, D. Ferrand, J. Cibert, Appl. Phys. Lett. 103, 222106 (2013)

33. W. Wardzynski, W. Giriat, H. Szymczak, R. Kowalczyk, Phys. Status Solidi B 49, 71 (1972)

34. Le Si Dang, J. Cibert, Y. Gobil, K. Saminadayar, S. Tatarenko, Appl. Phys. Lett. 55, 235 (1989)

35. D. Berlincourt, H. Jaffe, L.R. Shiozawa, Phys. Rev. 129, 1009 (1963)

36. J.M. Hartmann, J. Cibert, F. Kany, H. Mariette, M. Charleux, P. Alleyson, R. Langer, G. Feuillet, J. Appl. Phys. 80, 6257 (1996)

37. M.-E. Pistol, C.E. Pryor, Phys. Rev. B 78, 115319 (2008)

38. M. Altarelli, N.O. Lipari, Phys. Rev. B 15, 4898 (1977)

39. G. Fishman, Semi-conducteurs, les bases de la théorie k.p, Editions de l'Ecole polytechnique, Paris, 2010

40. T.E. Trammell, X. Zhang, Y. Li, L.-Q. Chen, E.C. Dickey, J. Cryst. Growth 310, 3084 (2008)

41. R.M. Martin, Phys. Rev. B 6, 4546 (1972)

42. D.A. Faux, J.R. Downes, E.P. O'Reilly, J. Appl. Phys. 82, 3754 (1997)

43. L.J. Zhang, M. d'Avezac, J.-W. Luo, A. Zunger, Nano Lett. 12, 984 (2012)

44. V. Schmidt, P.C. McIntyre, U. Gösele, Phys. Rev. B 77, $235302(2008)$

Open Access This article is distributed under the terms of the Creative Commons Attribution License http:// creativecommons.org/licenses/by/4.0 which permits unrestricted use, distribution, and reproduction in any medium, provided the original author(s) and source are credited. 


\section{Appendix}

\section{A Lamé-Clapeyron-Navier equations}

The full Lamé-Clapeyron-Navier equations are written for the three crystal structures and orientations.

\section{A.1 Wurzite, c-axis}

$$
\begin{gathered}
c_{11} \frac{\partial^{2} u_{x}}{\partial x^{2}}+\frac{c_{11}-c_{12}}{2} \frac{\partial^{2} u_{x}}{\partial y^{2}}+c_{44} \frac{\partial^{2} u_{x}}{\partial z^{2}} \\
+\frac{c_{11}+c_{12}}{2} \frac{\partial^{2} u_{y}}{\partial x \partial y}+\left(c_{13}+c_{44}\right) \frac{\partial^{2} u_{z}}{\partial x \partial z}=0 \\
\frac{c_{11}-c_{12}}{2} \frac{\partial^{2} u_{y}}{\partial x^{2}}+c_{11} \frac{\partial^{2} u_{y}}{\partial y^{2}}+c_{44} \frac{\partial^{2} u_{y}}{\partial z^{2}} \\
+\left(c_{13}+c_{44}\right) \frac{\partial^{2} u_{z}}{\partial y \partial z}+\frac{c_{11}+c_{12}}{2} \frac{\partial^{2} u_{x}}{\partial x \partial y}=0 \\
c_{44} \frac{\partial^{2} u_{z}}{\partial x^{2}}+c_{44} \frac{\partial^{2} u_{z}}{\partial y^{2}}+c_{33} \frac{\partial^{2} u_{z}}{\partial z^{2}} \\
+\left(c_{13}+c_{44}\right) \frac{\partial^{2} u_{x}}{\partial x \partial z}+\left(c_{13}+c_{44}\right) \frac{\partial^{2} u_{y}}{\partial y \partial z}=0 .
\end{gathered}
$$

\section{A.2 Zinc-blende, $\left\langle\begin{array}{lll}1 & 1 & 1\end{array}\right\rangle$ axis}

$$
\begin{aligned}
& \tilde{c}_{11} \frac{\partial^{2} u_{x}}{\partial x^{2}}+\frac{\tilde{c}_{11}-\tilde{c}_{12}}{2} \frac{\partial^{2} u_{x}}{\partial y^{2}}+\tilde{c}_{44} \frac{\partial^{2} u_{x}}{\partial z^{2}} \\
& \quad+\frac{\tilde{c}_{11}+\tilde{c}_{12}}{2} \frac{\partial^{2} u_{y}}{\partial x \partial y}+\left(\tilde{c}_{13}+\tilde{c}_{44}\right) \frac{\partial^{2} u_{z}}{\partial x \partial z} \\
& \quad+2 \tilde{c}_{14} \frac{\partial^{2} u_{x}}{\partial y \partial z}+2 \tilde{c}_{14} \frac{\partial^{2} u_{y}}{\partial z \partial x}+2 \tilde{c}_{14} \frac{\partial^{2} u_{z}}{\partial x \partial y}=0, \\
& \frac{\tilde{c}_{11}-\tilde{c}_{12}}{2} \frac{\partial^{2} u_{y}}{\partial x^{2}}+\tilde{c}_{11} \frac{\partial^{2} u_{y}}{\partial y^{2}}+\tilde{c}_{44} \frac{\partial^{2} u_{y}}{\partial z^{2}} \\
& \quad+\left(\tilde{c}_{13}+\tilde{c}_{44}\right) \frac{\partial^{2} u_{z}}{\partial y \partial z}+\frac{\tilde{c}_{11}+\tilde{c}_{12}}{2} \frac{\partial^{2} u_{x}}{\partial x \partial y} \\
& \quad+2 \tilde{c}_{14} \frac{\partial^{2} u_{x}}{\partial z \partial x}+\tilde{c}_{14} \frac{\partial^{2} u_{z}}{\partial x^{2}}-2 \tilde{c}_{14} \frac{\partial^{2} u_{y}}{\partial y \partial z}-\tilde{c}_{14} \frac{\partial^{2} u_{z}}{\partial y^{2}}=0 \\
& \tilde{c}_{44} \frac{\partial^{2} u_{z}}{\partial x^{2}}+\tilde{c}_{44} \frac{\partial^{2} u_{z}}{\partial y^{2}}+\tilde{c}_{33} \frac{\partial^{2} u_{z}}{\partial z^{2}} \\
& \quad+\left(\tilde{c}_{13}+\tilde{c}_{44}\right) \frac{\partial^{2} u_{x}}{\partial x \partial z}+\left(\tilde{c}_{13}+\tilde{c}_{44}\right) \frac{\partial^{2} u_{y}}{\partial y \partial z} \\
& \quad+2 \tilde{c}_{14} \frac{\partial^{2} u_{x}}{\partial x \partial y}+\tilde{c}_{14} \frac{\partial^{2} u_{y}}{\partial x^{2}}-\tilde{c}_{14} \frac{\partial^{2} u_{y}}{\partial y^{2}}=0,
\end{aligned}
$$

\section{A.3 Zinc-blende, $\left\langle\begin{array}{lll}0 & 0 & 1\end{array}\right\rangle$ axis}

$$
\begin{aligned}
& c_{11} \frac{\partial^{2} u_{x}}{\partial x^{2}}+c_{44} \frac{\partial^{2} u_{x}}{\partial y^{2}}+c_{44} \frac{\partial^{2} u_{x}}{\partial z^{2}} \\
& +\left(c_{12}+c_{44}\right) \frac{\partial^{2} u_{y}}{\partial x \partial y}+\left(c_{12}+c_{44}\right) \frac{\partial^{2} u_{z}}{\partial x \partial z}=0 \\
& c_{44} \frac{\partial^{2} u_{y}}{\partial x^{2}}+c_{11} \frac{\partial^{2} u_{y}}{\partial y^{2}}+c_{44} \frac{\partial^{2} u_{y}}{\partial z^{2}} \\
& +\left(c_{12}+c_{44}\right) \frac{\partial^{2} u_{z}}{\partial y \partial z}+\left(c_{12}+c_{44}\right) \frac{\partial^{2} u_{x}}{\partial x \partial y}=0, \\
& c_{44} \frac{\partial^{2} u_{z}}{\partial x^{2}}+c_{44} \frac{\partial^{2} u_{z}}{\partial y^{2}}+c_{11} \frac{\partial^{2} u_{z}}{\partial z^{2}} \\
& +\left(c_{12}+c_{44}\right) \frac{\partial^{2} u_{x}}{\partial x \partial z}+\left(c_{12}+c_{44}\right) \frac{\partial^{2} u_{y}}{\partial y \partial z}=0 .
\end{aligned}
$$

\section{B Generalized shear strains}

The present study involves Lamé-Clapeyron-Navier equations describing the response of a system which is invariant under a translation along the $z$-axis and isotropic in the basal $x y$ plane, to body forces which are periodic in a rotation around the $z$-axis: $F_{z}=F \sin 3 \theta$ for a zinc-blende NW along a $\langle 111\rangle$ axis, and $F_{r}=F \cos 4 \theta$, $F_{\theta}=-F \sin 4 \theta$ for a zinc-blende NW along a $\langle 0001\rangle$ axis. Note that $F_{z}=F \cos (\theta)$ over the whole structure describes a uniform axial shear strain applied to the system, and $F_{r}=F \sin 2 \theta, F_{\theta}=F \cos 2 \theta$ a uniform transverse shear strain: a transfer matrix method was proposed in reference [9] for multishell NWs submitted to these two types of shear strain. The present study involves similar body forces distributions with a faster dependence on $\theta$, localized in the shell: $F_{z}=F \sin p \theta$ with $p=3$, and $F_{r}=F \cos p \theta, F_{\theta}=-F \sin p \theta$ with $p=4$. Other orientations of the NWs will involve combinations of such body forces distributions.

We thus have to calculate a displacement field $\delta \mathbf{u}$, solution of the Lamé-Clapeyron-Navier equation:

$$
\begin{aligned}
& \frac{\hat{c}_{11}-\hat{c}_{12}}{2}\left(\frac{\partial^{2}}{\partial x^{2}}+\frac{\partial^{2}}{\partial y^{2}}\right) \delta u_{x} \\
& \quad+\frac{\hat{c}_{11}+\hat{c}_{12}}{2} \frac{\partial}{\partial x}\left(\frac{\partial \delta u_{x}}{\partial x}+\frac{\partial \delta u_{y}}{\partial y}\right)+F_{x}=0, \\
& \frac{\hat{c}_{11}-\hat{c}_{12}}{2}\left(\frac{\partial^{2}}{\partial x^{2}}+\frac{\partial^{2}}{\partial y^{2}}\right) \delta u_{y} \\
& \quad+\frac{\hat{c}_{11}+\hat{c}_{12}}{2} \frac{\partial}{\partial y}\left(\frac{\partial \delta u_{x}}{\partial x}+\frac{\partial \delta u_{y}}{\partial y}\right)+F_{y}=0,
\end{aligned}
$$




$$
\hat{c}_{44}\left(\frac{\partial^{2}}{\partial x^{2}}+\frac{\partial^{2}}{\partial y^{2}}\right) \delta u_{z}+F_{z}=0 .
$$

As the response of a linear, transversely isotropic system to an oscillating perturbation, the general solution is expected to show the same oscillatory behavior, in $\cos (p \theta)$ or $\sin p \theta$.

The boundary conditions are the continuity of the total displacement field, $\mathbf{u}+\delta \mathbf{u}$, at the interface, and that the stress components acting on the interface and on the sidewall surface $\left(\sigma_{r r}, \sigma_{r \theta}, \sigma_{r z}\right)$ all vanish. The last condition must be achieved for the total stress, corresponding to $\mathbf{u}+\delta \mathbf{u}$. For the displacement field, it is sufficient to write that the additional displacement field does not break the contact which has been established by the cylindrical displacement field, hence $\delta \mathbf{u}=0$. Note that the symmetry of the system and that of the shear strain strongly reduce the number of parameters to be determined from boundary conditions. For instance, the condition that the integral of $\sigma_{z z}$ vanishes is automatically preserved by the oscillating character of $\delta \mathbf{u}$.

\section{B.1 Axial shear strain and $\langle\mathbf{1} 11\rangle{ }^{N}$ NS}

In the absence of driving force in the basal plane, we keep $\delta u_{r}=0$ and $\delta u_{\theta}=0$, and look for $\delta u_{z}=\varphi(r) \sin p \theta$, with $\varphi(r)$ obeying equation (35). In cylindrical coordinates, that reads:

$$
\tilde{c}_{44}\left(\frac{\partial^{2}}{\partial r^{2}}+\frac{1}{r} \frac{\partial}{\partial r}+\frac{1}{r^{2}} \frac{\partial^{2}}{\partial \theta^{2}}\right)[\varphi(r) \sin p \theta]+F_{z}=0
$$

Or

$$
\tilde{c}_{44}\left(\frac{\mathrm{d}^{2}}{\mathrm{~d} r^{2}}+\frac{1}{r} \frac{\mathrm{d}}{\mathrm{d} r}-\frac{p^{2}}{r^{2}}\right) \varphi(r) \sin p \theta+F_{z}=0 .
$$

The general solution is the sum of functions $\sim r^{n}: n=-1$ provides a particular solution which compensates for $F_{z}$, and for $n= \pm p$, the sum of derivatives vanishes.

With $F_{z}=\tilde{c}_{14} 8 B_{s} r_{c}^{2} \sin 3 \theta / r^{3}$ in the shell, we obtain:

$$
\frac{\delta u_{z}}{r_{c}}=\left[\alpha_{3} \rho^{3}+\alpha_{-3} \rho^{-3}+\alpha_{-1} \rho^{-1}\right] \frac{\tilde{c}_{14}}{\tilde{c}_{44}} B_{s} \sin 3 \theta,
$$

where $\rho=r / r_{c}$, with $\alpha_{-1}^{s}=-1$ in the shell and $\alpha_{-1}^{c}=0$ in the core. Also, $\alpha_{-3}^{c}=0$ in the core to avoid a singularity at $r=0$. The additional strain is thus:

$$
\begin{aligned}
\delta \varepsilon_{r z} & =\frac{1}{2} \frac{\partial}{\partial r} \delta u_{z} \\
& =\left[3 \alpha_{3} \rho^{2}-3 \alpha_{-3} \rho^{-4}-\alpha_{-1} \rho^{-2}\right] \frac{\tilde{c}_{14}}{\tilde{c}_{44}} B_{s} \sin 3 \theta
\end{aligned}
$$

and

$$
\begin{aligned}
\sigma_{r z} & =2 \tilde{c}_{44} \delta \varepsilon_{r z}+\tilde{c}_{14} \sin 3 \theta\left(\epsilon_{r r}-\varepsilon_{\theta \theta}\right) \\
& =\left[3 \alpha_{3} \rho^{2}-3 \alpha_{-3} \rho^{-4}+\left(2-\alpha_{-1}\right) \rho^{-2}\right] \tilde{c}_{14} B_{s} \sin 3 \theta .
\end{aligned}
$$

The three remaining parameters $\alpha_{3}^{c}, \alpha_{3}^{s}$ and $\alpha_{-3}^{s}$ are determined by the non-trivial boundary conditions, on $u_{z}$ (at the interface) and $\sigma_{r z}$ (at the interface and surface). It is quite convenient to write these conditions using a transfer matrix:

$$
\begin{aligned}
\left(\begin{array}{c}
\left(\frac{\delta u_{z}}{r_{c}}\right) \\
\left(\frac{\sigma_{r z}}{\tilde{c}_{44}}\right)
\end{array}\right)= & \frac{\tilde{c}_{14}}{\tilde{c}_{44}} B_{s} \sin 3 \theta\left(\begin{array}{cc}
\rho^{3} & \rho^{-3} \\
3 \rho^{2} & -3 \rho^{-4}
\end{array}\right)\left(\begin{array}{c}
\alpha_{3} \\
\alpha_{-3}
\end{array}\right) \\
& +\alpha_{-1} \frac{\tilde{c}_{14}}{\tilde{c}_{44}} B_{s} \sin 3 \theta\left(\begin{array}{c}
\rho^{-1} \\
-3 \rho^{-2}
\end{array}\right) .
\end{aligned}
$$

At the interface $(\rho=1)$, if we omit the difference in stiffness coefficients between the two materials:

$$
\left(\begin{array}{cc}
1 & 1 \\
3 & -3
\end{array}\right)\left(\begin{array}{c}
\alpha_{3}^{c} \\
0
\end{array}\right)=\left(\begin{array}{cc}
1 & 1 \\
3 & -3
\end{array}\right)\left(\begin{array}{c}
\alpha_{3}^{s} \\
\alpha_{-3}^{s}
\end{array}\right)+\left(\begin{array}{c}
1 \\
-3
\end{array}\right)
$$

Or

$$
\left(\begin{array}{c}
\alpha_{3}^{c} \\
0
\end{array}\right)=\left(\begin{array}{c}
\alpha_{3}^{s} \\
\alpha_{-3}^{s}
\end{array}\right)+\left(\begin{array}{l}
0 \\
1
\end{array}\right)
$$

At the surface, using equation (B.2) at $r=r_{s}(\rho=1 / \sqrt{\eta})$, and keeping only the second component of the vectors (the stress which must be zero), we obtain:

$$
0=\left(\alpha_{3}^{s}-\eta^{3} \alpha_{-3}^{s}\right)-\eta^{2} .
$$

Hence $\alpha_{3}^{c}=\alpha_{3}^{s}=\eta^{2}(1-\eta)$ and $\alpha_{-3}^{s}=-1$.

If we assume a different hardness with a single factor $\chi$ between the stiffness coefficients of the shell with respect to those of the core material, equation (B.3) becomes:

$$
\left(\begin{array}{cc}
1 & 1 \\
3 & -3
\end{array}\right)\left(\begin{array}{c}
\alpha_{3}^{c} \\
0
\end{array}\right)=\left(\begin{array}{cc}
1 & 1 \\
3 \chi & -3 \chi
\end{array}\right)\left(\begin{array}{c}
\alpha_{3}^{s} \\
\alpha_{-3}^{s}
\end{array}\right)+\left(\begin{array}{c}
1 \\
-3 \chi
\end{array}\right),
$$

and the result is:

$$
\begin{aligned}
\alpha_{3}^{c} & =\eta^{2}(1-\eta) \frac{2 \chi}{1+\chi+\eta^{3}(1-\chi)} \\
\alpha_{3}^{s} & =\eta^{2}(1-\eta) \frac{1+\chi}{1+\chi+\eta^{3}(1-\chi)} \\
\alpha_{-3}^{s} & =-1-\eta^{2}(1-\eta) \frac{1-\chi}{1+\chi+\eta^{3}(1-\chi)} .
\end{aligned}
$$

The correction for $\chi$ non-unity is small for the actual NW configurations considered here: with $\chi=1.2$ and $\eta=0.2$, the corrective factor is $10 \%$ for $\alpha_{3}^{c}$ and negligible for the shell.

This result was used in the case of the $\langle 111\rangle$ core-shell NWs and it can be extended to multishell NWs.

\section{B.2 Transverse shear strain and $\left\langle\begin{array}{llll}0 & 0 & 1\end{array}\right\rangle$ NWs}

The problem is similar to the previous one: we have to find the response of a system with transverse isotropic character, to a body force distribution $\mathbf{F}$. The body forces $F$ represent an in-plane shear strain, with a four-fold symmetry due to the $\cos 4 \theta$ factor. A usual shear strain would have a $\cos 2 \theta$ and $\sin 2 \theta$ factors, as described in reference [9]. The solution is a bit more complex than the response to 
axial shear because we are dealing with a $2 \mathrm{D}$, not $1 \mathrm{D}$, problem.

The equation to be solved, equation (42), is, in polar coordinates:

$$
\begin{aligned}
& {\left[2(1-\nu)\left(\frac{\partial^{2}}{\partial r^{2}}+\frac{1}{r} \frac{\partial}{\partial r}-\frac{1}{r^{2}}\right)+(1-2 \nu) \frac{1}{r^{2}} \frac{\partial^{2}}{\partial \theta^{2}}\right] \delta u_{r}} \\
& +\left[\frac{1}{r} \frac{\partial^{2}}{\partial r \partial \theta}-(3-4 \nu) \frac{1}{r^{2}} \frac{\partial}{\partial \theta}\right] \delta u_{\theta}+\frac{2 F_{r}}{\hat{c}_{11}+\hat{c}_{12}}=0 \\
& {\left[\frac{1}{r} \frac{\partial^{2}}{\partial r \partial \theta}+(3-4 \nu) \frac{1}{r^{2}} \frac{\partial}{\partial \theta}\right] \delta u_{r}} \\
& +\left[(1-2 \nu)\left(\frac{\partial^{2}}{\partial r^{2}}+\frac{1}{r} \frac{\partial}{\partial r}-\frac{1}{r^{2}}\right)+2(1-\nu) \frac{1}{r^{2}} \frac{\partial^{2}}{\partial \theta^{2}}\right] \delta u_{\theta} \\
& +\frac{2 F_{\theta}}{\hat{c}_{11}+\hat{c}_{12}}=0 .
\end{aligned}
$$

The general solution can be written:

$$
\begin{aligned}
\frac{\delta u_{r}}{r_{c}} & =\frac{c}{\hat{c}_{11}+\hat{c}_{12}} B_{s} g_{r}(r) \cos 4 \theta \\
\frac{\delta u_{\theta}}{r_{c}} & =\frac{c}{\hat{c}_{11}+\hat{c}_{12}} B_{s} g_{\theta}(r) \sin 4 \theta .
\end{aligned}
$$

where $g_{r}$ and $g_{\theta}$ are two dimensionless functions which are sums of terms in $\rho^{n}$ with $\rho=r / r_{c}$ and the $n$ are integer (positive or negative).

For functions $\sim r^{n} \cos p \theta$ or $r^{n} \sin p \theta$, the derivative contributions in equation (B.3) vanish if $n^{2}=(p \pm 1)^{2}$. In the present case, $p=4$ hence $n= \pm 3, \pm 5$. In addition, the prefactors $\alpha_{n}$ and $\alpha_{n}$ of $r^{n}$ for a given value of $n$ are linked since the two equations of equation ( B.3) must be satisfied. Finally, the prefactors $\alpha_{-1}$ and $\alpha_{-1}^{\prime}$ are fully determined by the fact that it is the $r^{-1}$ contribution in $g_{r}$ and $g_{\theta}$ which makes equation (B.3) to be satisfied. As the $\alpha_{n}$ with negative indices all vanish in the core (to avoid a singularity at $r=0$ ), we have to determine six parameters, $\alpha_{3}^{c}$ and $\alpha_{5}^{c}$ in the core, $\alpha_{3}^{s}, \alpha_{-3}^{s}, \alpha_{5}^{s}$ and $\alpha_{-5}^{s}$ in the shell.

Boundary conditions are the continuity of $\delta \mathbf{u}$ at the interface, and the compensation of stress components acting on the interface and sidewall surface. The relevant stress components are, in the shell:

$$
\begin{aligned}
\sigma_{r r} & =\hat{c}_{11} \delta \varepsilon_{r r}+\hat{c}_{12} \delta \varepsilon_{\theta \theta}+\frac{c}{2} \cos 4 \theta \frac{\varepsilon_{r r}-\varepsilon_{\theta \theta}}{2} \\
& =\hat{c}_{11} \frac{\partial}{\partial r} \delta u_{r}+\hat{c}_{12}\left(\frac{\partial}{r \partial \theta} \delta u_{\theta}+\frac{\delta u_{r}}{r}\right)-\frac{c}{2} B_{s} \rho^{-2} \cos 4 \theta \\
\sigma_{r \theta} & =\hat{c}_{66} \varepsilon_{r \theta}=\hat{c}_{66} \frac{1}{2}\left(\frac{\partial}{r \partial \theta} \delta u_{r}+\frac{\partial}{\partial r} \delta u_{\theta}-\frac{\delta u_{\theta}}{r}\right) .
\end{aligned}
$$

They are similar in the core, but for $B_{c}=0$.

That makes six boundary conditions.

Writing the two stress components:

$$
\begin{aligned}
\frac{\sigma_{r \theta}}{\hat{c}_{66}} & =\frac{c}{\hat{c}_{11}+\hat{c}_{12}} B_{s} g_{r \theta}(r) \cos 4 \theta \\
\frac{\sigma_{r r}}{\hat{c}_{11}+\hat{c}_{12}} & =\frac{c}{\hat{c}_{11}+\hat{c}_{12}} B_{s} g_{r r}(r) \sin 4 \theta .
\end{aligned}
$$

the four functions $g_{r}, g_{\theta}, g_{r \theta}$ and $g_{r r}$ which are submitted to boundary conditions at the interface can be once again expressed in the frame of a transfer matrix treatment.

$$
\left(\begin{array}{c}
g_{r} \\
g_{\theta} \\
g_{r \theta} \\
g_{r r}
\end{array}\right)=\mathbf{M}(\rho)\left(\begin{array}{c}
\alpha_{3} \\
\alpha_{-3} \\
\alpha_{5} \\
\alpha_{-5}
\end{array}\right)+\mathbf{V}(\rho)
$$

where

$\mathbf{M}(1)=\left(\begin{array}{cccc}1 & (3-2 \nu) & (1+2 \nu) & 1 \\ -1 & 2 \nu & -2(2-\nu) & 1 \\ -3 & -6 & -10 & -5 \\ 3(1-2 \nu) & -9(1-2 \nu) & 5(1-2 \nu) & -5(1-2 \nu)\end{array}\right)$

$$
\mathbf{M}(\rho)=\left(\begin{array}{cccc}
1 & 0 & 0 & 0 \\
0 & 1 & 0 & 0 \\
0 & 0 & \rho^{-1} & 0 \\
0 & 0 & 0 & \rho^{-1}
\end{array}\right) \mathbf{M}(1)\left(\begin{array}{cccc}
\rho^{3} & 0 & 0 & 0 \\
0 & \rho^{-3} & 0 & 0 \\
0 & 0 & \rho^{5} & 0 \\
0 & 0 & 0 & \rho^{-5}
\end{array}\right)
$$

and

$$
\mathbf{V}(\rho)=\frac{1}{4(1-2 \nu)(1-\nu)}\left(\begin{array}{c}
2(1-\nu) \rho^{-1} \\
-(1-2 \nu) \rho^{-1} \\
-(3-2 \nu) \rho^{-2} \\
-4(1-2 \nu) \rho^{-2}
\end{array}\right)
$$

in the shell, and $\mathbf{V}(\rho)=0$ in the core.

At the interface $(\rho=1)$,

$$
\left(\begin{array}{c}
g_{r} \\
g_{\theta} \\
g_{r \theta} \\
g_{r r}
\end{array}\right)=\mathbf{M}(1)\left(\begin{array}{c}
\alpha_{3}^{c} \\
0 \\
\alpha_{5}^{c} \\
0
\end{array}\right)=\mathbf{M}(1)\left(\begin{array}{c}
\alpha_{3}^{s} \\
\alpha_{-3}^{s} \\
\alpha_{5}^{s} \\
\alpha_{-5}^{s}
\end{array}\right)+\mathbf{V}(1)
$$

and at the surface $(\rho=1 / \sqrt{\eta})$ :

$$
\left(\begin{array}{c}
g_{r} \\
g_{\theta} \\
g_{r \theta} \\
g_{r r}
\end{array}\right)=\mathbf{M}\left(\frac{1}{\sqrt{\eta}}\right)\left(\begin{array}{c}
\alpha_{3}^{s} \\
\alpha_{-3}^{s} \\
\alpha_{5}^{s} \\
\alpha_{-5}^{s}
\end{array}\right)+\mathbf{V}\left(\frac{1}{\sqrt{\eta}}\right)
$$

The right-hand side can be written, using equation (B.7):

$$
\mathbf{M}\left(\frac{1}{\sqrt{\eta}}\right)\left(\begin{array}{c}
\alpha_{3}^{c} \\
0 \\
\alpha_{5}^{c} \\
0
\end{array}\right)+\mathbf{V}\left(\frac{1}{\sqrt{\eta}}\right)-\mathbf{M}\left(\frac{1}{\sqrt{\eta}}\right) \mathbf{M}^{-1}(1) \mathbf{V}(1)
$$


The condition that and the stress at the interface vanishes implies that $\alpha_{3}^{s}$ and $\alpha_{5}^{s}$ are determined by equating the last two lines of equation (B.8) to zero.

The final result is:

$$
\begin{aligned}
\alpha_{3}^{c} & =\frac{-\nu+\eta^{2}\left[11-2 \nu-20 \eta+3 \eta^{2}(3+\nu)\right]}{12(1-\nu)(1-2 \nu)} \\
\alpha_{5}^{c} & =\frac{1-\eta^{3}\left[14-4 \nu-25 \eta+4 \eta^{2}(3+\nu)\right]}{40(1-\nu)(1-2 \nu)} \\
\alpha_{3}^{s} & =\frac{\eta^{2}\left[11-2 \nu-20 \eta+3 \eta^{2}(3+\nu)\right]}{12(1-\nu)(1-2 \nu)}
\end{aligned}
$$

$$
\begin{aligned}
\alpha_{-3}^{s} & =\frac{-5}{24(1-\nu)(1-2 \nu)} \\
\alpha_{5}^{s} & =\frac{-\eta^{3}\left[14-4 \nu-25 \eta+4 \eta^{2}(3+\nu)\right]}{40(1-\nu)(1-2 \nu)} \\
\alpha_{-5}^{s} & =\frac{3+\nu}{20(1-\nu)(1-2 \nu)} .
\end{aligned}
$$

Here we have assumed that the stiffness constants are the same in the core and in the shell. Different values of the stiffness constants can be accommodated by writing different matrices $\mathbf{M}^{c}$ and $\mathbf{M}^{s}$. And of course this transfer matrix method can be extended to multishell NWs. 